\title{
Effects of Reducing the South and Reinforcing the North Method on Inflammatory Injury Induced by Hyperlipidemia
}

\author{
Hongjin Wu $(\mathbb{D}$, Weiwei Dai, Libo Wang, Jie Zhang, and Chenglong Wang \\ Central Laboratory for Science and Technology, Longhua Hospital Shanghai University of Traditional Chinese Medicine, \\ Shanghai 200032, China
}

Correspondence should be addressed to Hongjin Wu; wuhj130707@shutcm.edu.cn

Received 11 June 2021; Revised 17 August 2021; Accepted 2 September 2021; Published 21 September 2021

Academic Editor: Arham Shabbir

Copyright (C) 2021 Hongjin Wu et al. This is an open access article distributed under the Creative Commons Attribution License, which permits unrestricted use, distribution, and reproduction in any medium, provided the original work is properly cited.

Inflammation is the pathophysiological basis of hyperlipidemia-related disease (HRD). Reducing the south and reinforcing the north method (RSRN) has a positive effect on HRD. However, the pharmacological mechanisms of RSRN are still unclear in the treatment of HRD. We obtained RSRN compounds from the Traditional Chinese Medicine Systems Pharmacology (TCMSP) and identified potential targets of these compounds through target fishing based on the TCMSP databases. Next, we identified the HRD targets by using multiple databases. Then, the overlapping genes between the RSRN potential targets and the HRD targets were used to establish a protein-protein interaction (PPI) network, and we further analyzed their interactions and identified the major hub genes in this network. Subsequently, the Metascape database was utilized to conduct the enrichment of Gene Ontology biological processes (GO) and Kyoto Encyclopedia of Genes and Genomes (KEGG) pathways. A total of 187 potential active components and 106 related core targets were obtained and identified overall. Then after the Metascape enrichment analysis, a total of 148 KEGG pathways were screened, which were mainly associated with AGE-RAGE signaling pathway, PI3K-Akt signaling pathway, TNF signaling pathway, and NF-kappa B signaling pathway. Furthermore, 34 hub genes, such as AKT1, NF$\kappa \mathrm{Bp} 65$ (RELA), I $\kappa \mathrm{B} \alpha(\mathrm{CHUK}), \mathrm{MAPK} 8$, and MAPK14, CCND1, were considered potential therapeutic targets. Furthermore, evaluations of protein levels of NF- $\kappa \mathrm{Bp} 65, \mathrm{I} \kappa \mathrm{B} \alpha$, TNF- $\alpha$, IL- $1 \beta$, and IL- 6 were performed for experimental validation. RSRN can reduce the expression of NF- $\kappa \mathrm{Bp} 65$ protein, increase the level of $\mathrm{I} \kappa \mathrm{B} \alpha$ protein, and reduce the protein levels of TNF- $\alpha$, IL- $1 \beta$, and IL-6 in ovariectomized rats. The results indicate that the mechanism of RSRN against inflammation may be related to AKT1, NF$\kappa \mathrm{Bp} 65, \mathrm{I} \kappa \mathrm{B} \alpha, \mathrm{MAPK} 8$, and MAPK14, as well as TNF, NF-kappa B, PI3K-Akt signaling pathways.

\section{Introduction}

The decreased ovarian function and estrogen levels in menopausal women contributes to the increase in the prevalence of dyslipidemia, osteoporosis, and urinary tract infection, of which hyperlipidemia is the most insidious [1]. Previous studies have reported that hyperlipidemia could lead to atherosclerosis (AS), coronary heart disease (CHD), and Alzheimer's disease $[2,3]$. There should be necessary interventions to be done for preventing the hyperlipidemia related diseases (HRD), thus to reduce the incidence rate and mortality of AS and CHD in middle-aged and elderly women.

Traditional Chinese medicine (TCM) believes that the kidney is the congenital life basis. When women are in the menopausal period, kidney-Qi declines; Tiangui will be exhausted; Chong and Ren become deficient; essence and blood become insufficient; all these conditions can lead to imbalance between Yin and Yang and Zang-fu organs disorders, resulting in menopausal syndromes and hyperlipidemia-related diseases [4]. The pathogenesis of HRD belongs to the syndrome of deficiency of origin and excess of standard, while kidney Yin deficiency is the foundation, and heart fire is excessive, and essence deficiency and blood stasis is the standard [5]. In clinic, method of nourishing kidney and clearing heart can effectively alleviate and control these syndromes. Therefore, reducing the south (clearing heart) and reinforcing the north (nourishing kidney essence) may be the foundation of the HRD treatment. Reducing the south and reinforcing the north formula (RSRN) consist of eight 
herbs: Epimedium sagittatum (Siebold \&Zucc.) Maxim. (ES); Curculigo orchioides Gaerth. (CO); Angelica sinensis (Oliv.) Diels. (AS); Phellodendron chinense Schneid. (PC); Anemarrhena asphodeloides Bge. (AR); Morinda officinalis How (MO); Radix Salvia (RS); and Coptidis Rhizoma (CR). Pharmacological studies on RSRN have assessed its regulation of the sex hormone and lipid metabolism and its prevention of the atherosclerosis and cardiovascular disease during the climacteric period $[6,7]$. It was reported [8,9] that Modified RSRN could enhance the myocardial microvascular density, improve the endothelial secretion function and hemorheology, and regulate the endocrine system and lipid metabolism in ovariectomized rats.

Network pharmacology is a kind of new method for investigating the pharmacological mechanisms of TCM based on system biology and multidirectional pharmacology [10]. Due to the complexity of the TCM components and the uncertainty of their targets, conventional pharmacology research methods are difficult to fully elucidate the potential molecular mechanism of TCM compound in the treatment of diseases. In this study, we dissected the mechanisms of RSRN in treating hyperlipidemia and atherosclerosis and identified compounds related to the RSRN with the help of network pharmacology method based on multiple databases; we obtained the compounds potential targets via target fishing and verified the results through experiment. The detailed procedures can be seen in Figure 1.

\section{Materials and Methods}

2.1. Identification of Main Active Components and Related Targets. Herbs were first confirmed by the comprehensive database of traditional Chinese medicine (TCMID), http:// 119.3.41.228: 8000/TCMID/prescription search/). Then all of the constituent data of RSRN were obtained from the TCMSP database (http://lsp.nwu.edu.cn/tcmsp.php) [11]. The active components were further identified using the parameters of the oral bioavailability (OB); we set the threshold of $\mathrm{OB}$ at $\geq 30 \%$ and the drug likeness (DL) at $\geq 0.18$ [12]. Then we used the TCMSP platform to predict the targets of active ingredients. The target information was obtained by correcting all the retrieved targets to their official names (official symbol) based on the UniprotKB search function in the protein database (UniProt) (http://www. uniprot.org/).

2.2. Disease Targets Identification by Multiple Databases. "Hyperlipidemia," "atherosclerosis," and "Alzheimer's disease" were selected as the key words for the retrieval of disease targets from the GeneCards database (https://www. genecards.org/), TTD database (Therapeutic Target Database, http://bidd.nus.edu.sg/group/cjttd/), DrugBank database (https://www.durgbank.ca/), OMIM database (Online Mendelian Inheritance in Man (https://www/omim.org/), and then the acquired disease targets were intersected by FunRich3.1.3 software. GSE57691 was screened and download from the GEO database (gene expression omnibus, https://www. ncbi. nlm. nih.gov/geo/) with "hyperlipidemia" and "atherosclerosis" as the key words, including 58 cases of disease group (49 cases of abdominal aortic aneurysm and 9 cases of aortic occlusive disease) and 10 cases of normal control group. We merged the above results and deleted the duplicate genes to obtain the final disease target genes by $R$ 4.0.2 software.

2.3. Construction and Analysis of Drug-Disease Target Network. Based on previous steps, drug-disease crossover genes were screened with $R$ software using the Venn Diagram package. The String 11.0 database (http://string-db. org/) was used to analyze the intersecting protein-protein interactions (PPIs) and then Cytoscape3.6.0 was used to determine the drug-disease target network. The "centiscape" plug-in was used to calculate the degree of freedom of drugdisease target. The higher the "degree" value, the greater the probability of playing the main function [13]. Using the Bisogenet and CytoNCA plug-ins of Cytoscape software (version 3.6.0), we set the parameters of degree centrality $(\mathrm{DC}>61)$ and the topology intermediateness $(\mathrm{BC}>600)$ and constructed the core target gene topology network; furthermore, we screened out the important candidate genes.

2.4. GO and KEGG Pathway Analysis. The screened drugdisease targets were evaluated by functional enrichment analyses. GO analysis was performed in three categories, namely biological processes (BP), cellular component (CC), and molecular function (MF), and the KEGG signaling pathway analysis was performed with $R$ software using the Bioconductor package. The KEGG pathway was introduced into Cytoscape 3.6.0. According to the degree value, the enrichment degree of pathway and gene was displayed, and the network diagram was drawn.

2.5. Herbal Preparation. We prepared RSRN using the following constituents: $30 \mathrm{~g}$ of Curculigo orchioides Gaertn (Xian Mao), $30 \mathrm{~g}$ of Epimedii Folium (Yin yang huo), $15 \mathrm{~g}$ of Morindae officinalis Radix (Ba ji tian), $15 \mathrm{~g}$ of Angelicae Sinensis Radix (Dang gui), $12 \mathrm{~g}$ of Phellodendron chinense Cortex (Huang bo), $10 \mathrm{~g}$ of Anemarrhenae Rhizoma (Zhi $\mathrm{mu}$ ), $12 \mathrm{~g}$ of Salviae Miltiorrhizae Radix et Rhizoma (Dan shen), and $6 \mathrm{~g}$ of Coptidis Rhizoma (Huang lian). We obtained all herbs from Longhua Hospital Shanghai University of TCM, China. The herbs were mixed, soaked in water for $0.5 \mathrm{~h}$, and decocted for $1 \mathrm{~h}$ in $5 \% \mathrm{v} / \mathrm{w}$ distilled $\mathrm{H}_{2} \mathrm{O}$ at $100^{\circ} \mathrm{C}$. Subsequently, the filtrate was collected, and the residue decocted for another hour with $5 \% \mathrm{v} / \mathrm{w}$ distilled water. Next, the filtrate was concentrated (RE-3000B, Ya-rong Biochemical Instrument Shanghai Co., Ltd) and lyophilized (LGJ-10D, Four-ring Science Instrument Plant Beijing Co., Ltd), and the resulting RSRN powder was kept at $-20^{\circ} \mathrm{C}$ until use. HPLC was used to determine the components in RSRN, and determination of curculigoside, Dihydrotanshinoen I, Icarisid I, Mangiferin, Sarsasapogenin, Jatrorrhizine in RSRN is shown in Figure 2. 


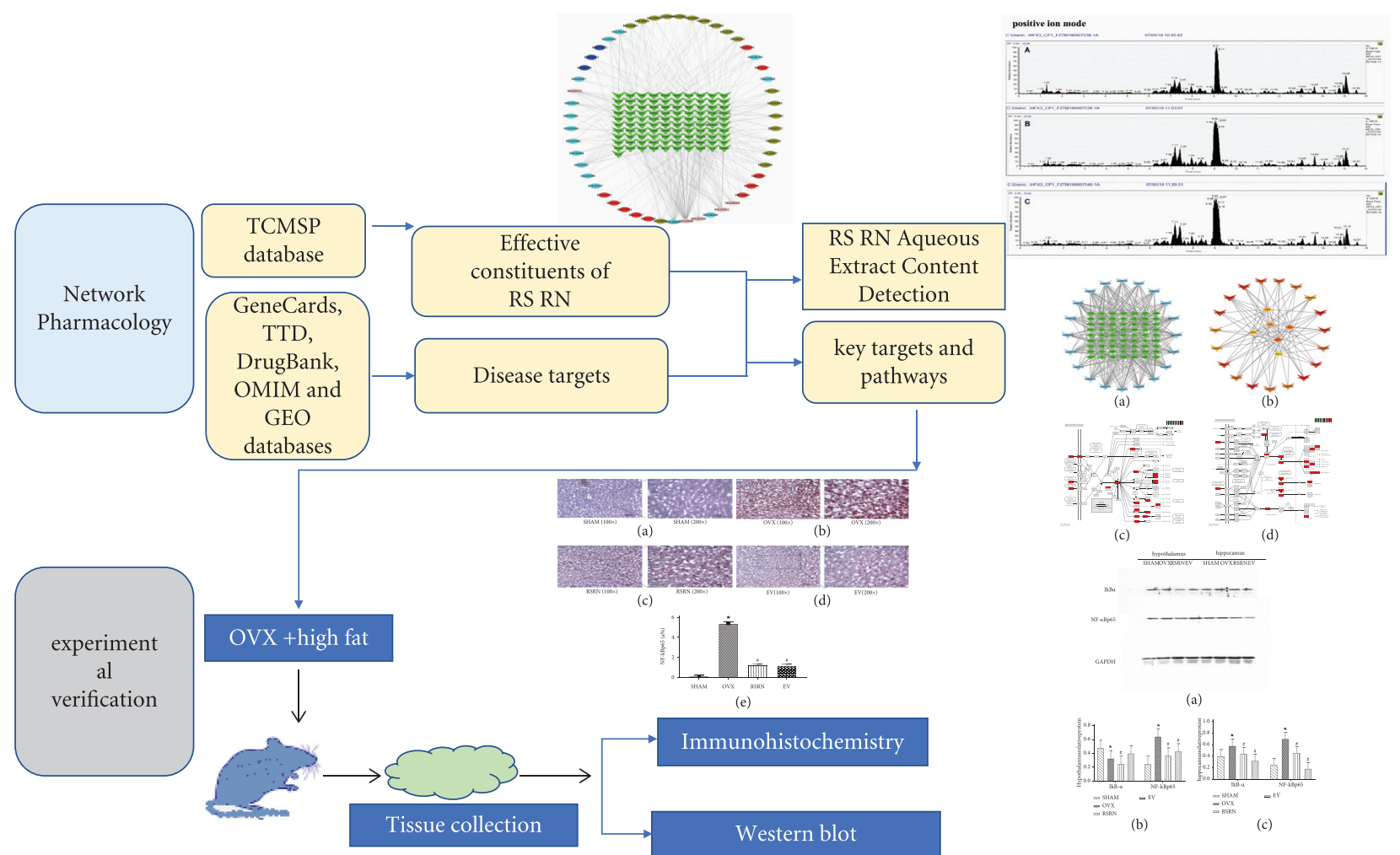

Figure 1: The detailed flowchart of the current study.

2.6. Animals and Administration. We obtained 40 healthy eight-week-old female Sprague-Dawley (SD) rats (mean weight: $200 \pm 20 \mathrm{~g}$ ) from Shanghai Slack Laboratory Animals Co., Ltd. [license number: SCXK (Hu) 2012-0002]. All the rats were housed in an air-conditioned room with $12 \mathrm{~h}$ lightdark cycles at a constant temperature $\left(22-26^{\circ} \mathrm{C}\right)$ and humidity $(50 \% \pm 10 \%)$. Further, the rats were provided with rodent chow and tap water ad libitum. The animal model of climacteric atherosclerosis was established by bilateral ovariectomy and high-fat diet [14]. After acclimation for one week, the rats underwent either ovariectomy $(n=30)$ or sham operation $(n=10)$ under anesthesia using an intraperitoneal injection of $30 \mathrm{mg} / \mathrm{kg}$ pentobarbital sodium. Nine days after surgery, we randomly divided 30 OVX rats into the following three groups: OVX (high-fat emulsion [15] with an equal volume of $\mathrm{ddH}_{2} \mathrm{O} ; 1 \mathrm{~mL} / 100 \mathrm{~g} /$ day), RSRN (high-fat emulsion with RSRN; $5.46 \mathrm{~g} / \mathrm{kg} /$ day), and $\mathrm{EV}$ groups (high-fat emulsion with estrogen valerate; $0.1 \mathrm{mg} / \mathrm{kg} /$ day). The sham-operated rats (SHAM group) received a normal diet with an equal volume of $\mathrm{ddH}_{2} \mathrm{O} ; 1 \mathrm{~mL} / 100 \mathrm{~g} /$ day. During the experimental period, the rats underwent weekly weight measurements. All procedures were approved by the Department of Laboratory Animal Science Longhua Hospital Shanghai University of TCM. The animal welfare and experimental procedures were conducted in strict accordance with the guidelines for the care and use of experimental animals and the ethics of Shanghai University of TCM. For the next six weeks after the last administration, the rats were food- and water deprived for $12 \mathrm{~h}$. The rats were anaesthetized by diethyl ether inhalation. The brain and the blood were taken for further use.
2.7. Immunohistochemistry Analysis. Immunohistrochemistry procedure was performed as previously reported protocol [16]. The sections were incubated with the primary antibody for NF- $\kappa \mathrm{B}$ p65 overnight at $4^{\circ} \mathrm{C}(\# 8242 ; 1: 100$, Cell Signalling Technology, MA,USA). After washing, the sections were incubated with the secondary antibody (\#MR-R100; MR Biotech, Shanghai, China) for $1 \mathrm{~h}$ and then stained with 3,3'-diaminobenzidine (DAB) (Biyuntian Institute of Biotechnology, Jiangsu, China). Signals were visualized by light microscopic observation. The results were analyzed by using Image $J$ software version $1.50 \mathrm{i}$ (National Institute of Health, USA). The investigators used the software to measure the ratio of positive area $(\mathrm{A} \%)$.

2.8. Western Blotting Analysis. The protein samples were separated by $10 \%$ SDS-PAGE transferred to PVDF membrane. The membrane was blocked with $1 \%$ BSA in TBST for $30 \mathrm{~min}$, the primary antibodies including NF- $\kappa$ Bp65 (\#8242; $1: 1000$; Cell Signalling Technology, MA, USA), I $\kappa \mathrm{B} \alpha$ (\#4812; 1:1000; Cell Signalling Technology, MA, USA), TNF- $\alpha$ (\#sc-52746; 1:1000; Santa Curz Biptechnology, USA), IL-1 $\beta$ (\#sc-52012; 1 : 1000; Santa Curz Biptechnology, USA), IL-6 (\#sc-32296; 1 : 1000; Santa Curz Biptechnology, USA) were incubated at $4^{\circ} \mathrm{C}$ overnight $(\geq 12 \mathrm{~h})$, and secondary antibodies for GAPDH (\#5174T; 1:5000; Cell Signalling Technology, MA, USA) and m-IgGk BP-HRP (\#sc516101; 1 : 5000; Santa Curz Biptechnology, USA) were used as the internal reference antibodies. Washed with TBST $5 \mathrm{~min} \times 3$ times, chemiluminescence imaging was performed with ECL luminescent liquid. 


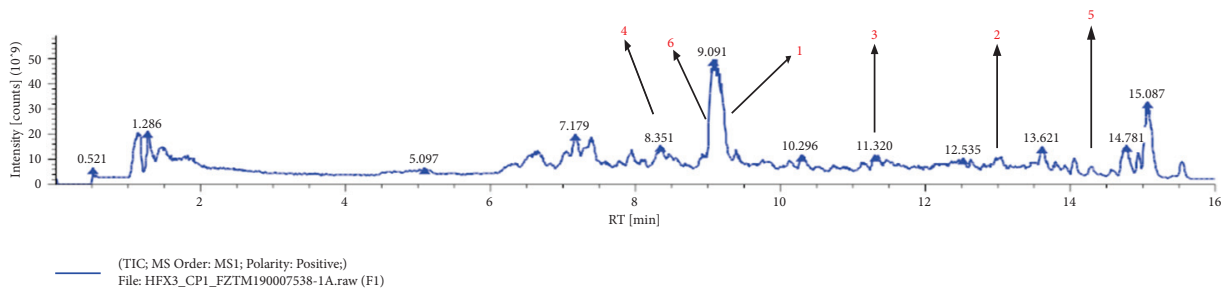

(a)
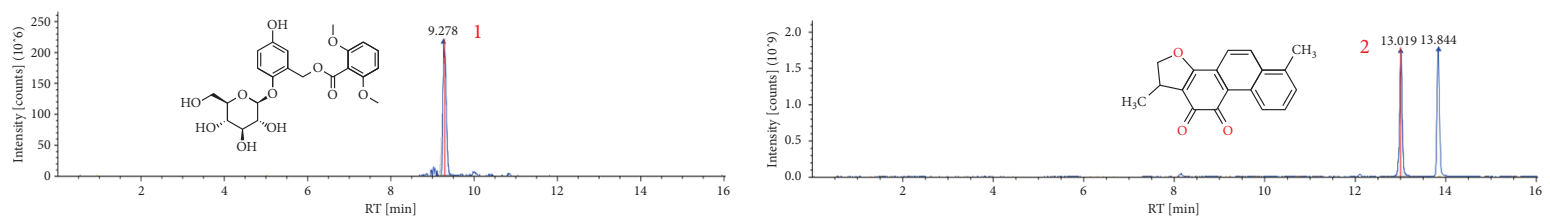

MW: 488.12923
File: HFX3_CP_blank.raw (F1) FTMS (+) MS

MW: 488.12923
File: HFX3_CP1_FZTM190007538-1A.raw (F2) FTMS (+) MS1
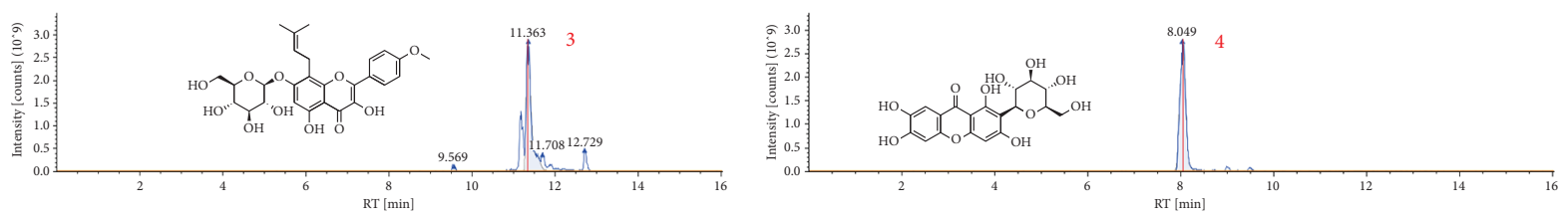

MW: 530.17870
File: HFX3_CP_blank.raw (F1) FTMS (+) MS

MW: 530.17870
File: HFX3_CP1_FZTM190007538-1A.raw (F2) FTMS (+) MS1
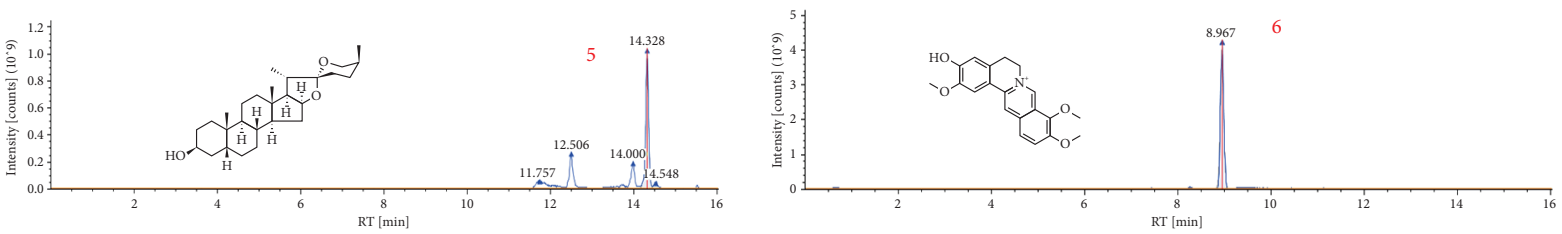

MW: 416.32869

(F) FTMS (+) MS1

- MW: 416.32869

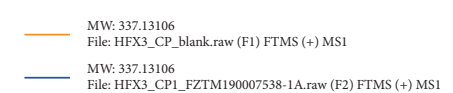

(b)

FIGURE 2: HPLC-MS mass specterometry analysis of the RSRN. (a) First-order spectrogram positive ion mode. (b) Second-order spectrogram positive ion mode. (c) Mass spectrometry of the main components; 1. Curculigoside; 2. Dihydrotanshinoen I; 3 . Icarisid I; 4. Mangiferin; 5. Sarsasapogenin; 6. Jatrorrhizine.

2.9. Statistical Analyses. Using SPSS20.0 statistical analysis software, the data were expressed as means \pm standard deviation (SD). The significant difference was expressed as $p<0.05$. One-way ANOVA was used to compare the experimental results among groups. LSD test (meeting the requirement of homogeneity of variance) or Dunnett's T3 (not meeting the requirement of homogeneity of variance) was used for further pairwise comparison.

\section{Results}

3.1. Active Components of RSRN. A total of 187 active components were identified from the TCMSP databases by the ADME thresholds (OB $\geq 30 \%, \mathrm{DL} \geq 0.18)$, including 8 components of Curculigo orchioides Gaertn (Xian Mao), 22 components of Epimedii Folium (Yin yang huo), 21 components of Morindae officinalis Radix (Ba ji tian), 2 components of Angelicae Sinensis Radix (Dang gui), 38 components of Phellodendron chinense Cortex (Huang bo), 17 components of Anemarrhenae Rhizoma (Zhi mu), 65 components of Salviae Miltiorrhizae Radix et Rhizoma (Dan shen), and 14 components of Coptidis Rhizoma (Huang lian) (Table 1).

3.2. Analysis of "RSRN-Compound-Target" Network. We conducted target fishing for these 187 active ingredients using the TCMSP databases based on chemical similarity and obtained 225 related targets. We evaluated the relationships between the components and targets with a constructed "RSRN-compound-target" network, which had a total of 365 nodes and 4841 edges (Figure 3(a)). The inverted triangles represent the active compounds, and the circles represent their targets. The network topology was analyzed by using centiscape plug-in, and the degree value of the topological network was 26.53 , the betweenness value was 469.26, the closeness value was 0.0012 , and the 
TABLE 1: Detailed information on 106 active compounds from RSRN.

\begin{tabular}{|c|c|c|c|c|}
\hline Mol ID & Components & $\mathrm{OB} \%$ & DL & Herbs \\
\hline MOL001506 & Supraene & 33.6 & 0.42 & $\mathrm{Ba}$ ji tian \\
\hline MOL002879 & Diop & 43.6 & 0.39 & $\mathrm{Ba}$ ji tian \\
\hline MOL002883 & Ethyl oleate (NF) & 32.4 & 0.19 & $\mathrm{Ba}$ ji tian \\
\hline MOL000358 & Beta-sitosterol & 36.9 & 0.75 & $\mathrm{Ba}$ ji tian \\
\hline MOL000359 & Sitosterol & 36.9 & 0.75 & $\mathrm{Ba}$ ji tian \\
\hline MOL006147 & Alizarin-2-methylether & 32.8 & 0.21 & $\mathrm{Ba}$ ji tian \\
\hline MOL009495 & 2-Hydroxy-1,5-dimethoxy-6-(methoxymethyl)-9,10-anthraquinone & 95.9 & 0.37 & $\mathrm{Ba}$ ji tian \\
\hline MOL009496 & 1,5,7-Trihydroxy-6-methoxy-2-methoxymethylanthracenequinone & 80.4 & 0.38 & Ba ji tian \\
\hline MOL009500 & 1,6-Dihydroxy-5-methoxy-2-(methoxymethyl)-9,10-anthraquinone & 105 & 0.34 & $\mathrm{Ba}$ ji tian \\
\hline MOL009503 & 1-Hydroxy-3-methoxy-9,10-anthraquinone & 104 & 0.21 & $\mathrm{Ba}$ ji tian \\
\hline MOL009504 & 1-Hydroxy-6-hydroxymethylanthracenequinone & 81.8 & 0.21 & $\mathrm{Ba}$ ji tian \\
\hline MOL009513 & 2-Hydroxy-1,8-dimethoxy-7-methoxymethylanthracenequinone & 112 & 0.37 & Ba ji tian \\
\hline MOL009519 & $(2 \mathrm{R}, 3 \mathrm{~S})-(+)-3^{\prime}, 5$-dihydroxy-4,7-dimethoxydihydroflavonol & 77.2 & 0.33 & Ba ji tian \\
\hline MOL009524 & 3beta,20(R),5-alkenyl-stigmastol & 36.9 & 0.75 & Ba ji tian \\
\hline MOL009525 & 3beta-24S(R)-butyl-5-alkenyl-cholestol & 35.4 & 0.82 & Ba ji tian \\
\hline MOL009537 & Americanin A & 46.7 & 0.35 & $\mathrm{Ba}$ ji tian \\
\hline MOL009541 & Asperuloside tetraacetate & 45.5 & 0.82 & Ba ji tian \\
\hline MOL009551 & Isoprincepin & 49.1 & 0.77 & Ba ji tian \\
\hline MOL009558 & 2-Hydroxyethyl 5-hydroxy-2-(2-hydroxybenzoyl)-4-(hydroxymethyl)benzoate & 62.3 & 0.26 & Ba ji tian \\
\hline MOL009562 & Ohioensin-A & 38.1 & 0.76 & $\mathrm{Ba}$ ji tian \\
\hline MOL000358 & Beta-sitosterol & 36.9 & 0.75 & Dang gui \\
\hline MOL000449 & Stigmasterol & 43.8 & 0.76 & Dang gui \\
\hline MOL001454 & Berberine & 36.9 & 0.78 & Huang bo \\
\hline MOL001458 & Coptisine & 30.7 & 0.86 & Huang bo \\
\hline MOL002636 & Kihadalactone A & 34.2 & 0.82 & Huang bo \\
\hline MOL013352 & Obacunone & 43.3 & 0.77 & Huang bo \\
\hline MOL002641 & Phellavin_qt & 35.9 & 0.44 & Huang bo \\
\hline MOL002643 & Delta 7-stigmastenol & 37.4 & 0.75 & Huang bo \\
\hline MOL002644 & Phellopterin & 40.2 & 0.28 & Huang bo \\
\hline MOL002651 & Dehydrotanshinone II A & 43.8 & 0.4 & Huang bo \\
\hline MOL002652 & delta7-dehydrosophoramine & 54.5 & 0.25 & Huang bo \\
\hline MOL002656 & Dihydroniloticin & 36.4 & 0.81 & Huang bo \\
\hline MOL002659 & Kihadanin A & 31.6 & 0.7 & Huang bo \\
\hline MOL002660 & Niloticin & 41.4 & 0.82 & Huang bo \\
\hline MOL002662 & Rutaecarpine & 40.3 & 0.6 & Huang bo \\
\hline MOL002663 & Skimmianin & 40.1 & 0.2 & Huang bo \\
\hline MOL002666 & Chelerythrine & 34.2 & 0.78 & Huang bo \\
\hline MOL000449 & Stigmasterol & 43.8 & 0.76 & Huang bo \\
\hline MOL002668 & Worenine & 45.8 & 0.87 & Huang bo \\
\hline MOL002670 & Cavidine & 35.6 & 0.81 & Huang bo \\
\hline MOL002671 & Candletoxin A & 31.8 & 0.69 & Huang bo \\
\hline MOL002672 & Hericenone $\mathrm{H}$ & 39 & 0.63 & Huang bo \\
\hline MOL002673 & Hispidone & 36.2 & 0.83 & Huang bo \\
\hline MOL000358 & Beta-sitosterol & 36.9 & 0.75 & Huang bo \\
\hline MOL000622 & Magnograndiolide & 63.7 & 0.19 & Huang bo \\
\hline MOL000762 & Palmidin A & 35.4 & 0.65 & Huang bo \\
\hline MOL000785 & Palmatine & 64.6 & 0.65 & Huang bo \\
\hline MOL000787 & Fumarine & 59.3 & 0.83 & Huang bo \\
\hline MOL000790 & Isocorypalmine & 35.8 & 0.59 & Huang bo \\
\hline MOL000098 & Quercetin & 46.4 & 0.28 & Huang bo \\
\hline MOL001131 & Phellamurin_qt & 56.6 & 0.39 & Huang bo \\
\hline MOL001455 & (S)-canadine & 53.8 & 0.77 & Huang bo \\
\hline MOL001771 & Poriferast-5-en-3beta-ol & 36.9 & 0.75 & Huang bo \\
\hline MOL001601 & 1,2,5,6-Tetrahydrotanshinone & 38.75 & 0.36 & Dan shen \\
\hline MOL001659 & Poriferasterol & 43.83 & 0.76 & Dan shen \\
\hline MOL001771 & Poriferast-5-en-3beta-ol & 36.91 & 0.75 & Dan shen \\
\hline MOL001942 & Isoimperatorin & 45.46 & 0.23 & Dan shen \\
\hline MOL002222 & Sugiol & 36.11 & 0.28 & Dan shen \\
\hline MOL002651 & Dehydrotanshinone II A & 43.76 & 0.4 & Dan shen \\
\hline MOL002776 & Baicalin & 40.12 & 0.75 & Dan shen \\
\hline
\end{tabular}


TABle 1: Continued.

\begin{tabular}{|c|c|c|c|c|}
\hline Mol ID & Components & $\mathrm{OB} \%$ & DL & Herbs \\
\hline MOL000569 & Digallate & 61.85 & 0.26 & Dan shen \\
\hline MOL000006 & Luteolin & 36.16 & 0.25 & Dan shen \\
\hline MOL006824 & $\alpha$-Amyrin & 39.51 & 0.76 & Dan shen \\
\hline MOL007036 & 5,6-Dihydroxy-7-isopropyl-1,1-dimethyl-2,3-dihydrophenanthren-4-one & 33.77 & 0.29 & Dan shen \\
\hline MOL007041 & 2-Isopropyl-8-methylphenanthrene-3,4-dione & 40.86 & 0.23 & Dan shen \\
\hline MOL007045 & $3 \alpha$-Hydroxytanshinone?a & 44.93 & 0.44 & Dan shen \\
\hline MOL007048 & (E)-3-[2-(3,4-dihydroxyphenyl)-7-hydroxy-benzofuran-4-yl]acrylic acid & 48.24 & 0.31 & Dan shen \\
\hline MOL007049 & 4-Methylenemiltirone & 34.35 & 0.23 & Dan shen \\
\hline MOL007050 & $\begin{array}{c}\text { 2-(4-Hydroxy-3-methoxyphenyl)-5-(3-hydroxypropyl)-7-methoxy-3- } \\
\text { benzofurancarboxaldehyde }\end{array}$ & 62.78 & 0.4 & Dan shen \\
\hline MOL007051 & 6-o-Syringyl-8-o-acetyl shanzhiside methyl ester & 46.69 & 0.71 & Dan shen \\
\hline MOL007058 & Formyltanshinone & 73.44 & 0.42 & Dan shen \\
\hline MOL007059 & 3-Beta-hydroxymethyllenetanshiquinone & 32.16 & 0.41 & Dan shen \\
\hline MOL007061 & Methylenetanshinquinone & 37.07 & 0.36 & Dan shen \\
\hline MOL007063 & Przewalskin a & 37.11 & 0.65 & Dan shen \\
\hline MOL007064 & Przewalskin b & 110.32 & 0.44 & Dan shen \\
\hline MOL007068 & Przewaquinone B & 62.24 & 0.41 & Dan shen \\
\hline MOL007069 & Przewaquinone c & 55.74 & 0.4 & Dan shen \\
\hline MOL007070 & (6S,7 R)-6,7-dihydroxy-1,6-dimethyl-8,9-dihydro-7h-naphtho[8,7-g] benzofuran-10,11-dione & 41.31 & 0.45 & Dan shen \\
\hline MOL007071 & Przewaquinone $\mathrm{f}$ & 40.31 & 0.46 & Dan shen \\
\hline MOL007077 & Sclareol & 43.67 & 0.21 & Dan shen \\
\hline MOL007079 & Tanshinaldehyde & 52.47 & 0.45 & Dan shen \\
\hline MOL007081 & Danshenol B & 57.95 & 0.56 & Dan shen \\
\hline MOL007082 & Danshenol A & 56.97 & 0.52 & Dan shen \\
\hline MOL007085 & Salvilenone & 30.38 & 0.38 & Dan shen \\
\hline MOL007088 & Cryptotanshinone & 52.34 & 0.4 & Dan shen \\
\hline MOL007093 & Dan-shexinkum d & 38.88 & 0.55 & Dan shen \\
\hline MOL007094 & Danshenspiroketallactone & 50.43 & 0.31 & Dan shen \\
\hline MOL007098 & Deoxyneocryptotanshinone & 49.4 & 0.29 & Dan shen \\
\hline MOL007100 & Dihydrotanshinlactone & 38.68 & 0.32 & Dan shen \\
\hline MOL007101 & Dihydrotanshinone I & 45.04 & 0.36 & Dan shen \\
\hline MOL007105 & Epidanshenspiroketallactone & 68.27 & 0.31 & Dan shen \\
\hline MOL002331 & N-Methylflindersine & 32.4 & 0.18 & Huang bo \\
\hline MOL002894 & Berberrubine & 35.7 & 0.73 & Huang bo \\
\hline MOL005438 & Campesterol & 37.6 & 0.71 & Huang bo \\
\hline MOL006392 & Dihydroniloticin & 36.4 & 0.82 & Huang bo \\
\hline MOL006401 & Melianone & 40.5 & 0.78 & Huang bo \\
\hline MOL006413 & Phellochin & 35.4 & 0.82 & Huang bo \\
\hline MOL006422 & Thalifendine & 44.4 & 0.73 & Huang bo \\
\hline MOL001607 & ZINC03982454 & 36.9 & 0.76 & Xian Mao \\
\hline MOL003578 & Cycloartenol & 38.7 & 0.78 & Xian Mao \\
\hline MOL000358 & Beta-sitosterol & 36.9 & 0.75 & Xian Mao \\
\hline MOL004114 & $3,2^{\prime}, 4^{\prime}, 6^{\prime}$-tetrahydroxy- $4,3^{\prime}$-dimethoxy chalcone & 52.7 & 0.28 & Xian Mao \\
\hline MOL004125 & Curculigoside B_qt & 83.4 & 0.19 & Xian Mao \\
\hline MOL004146 & Curculigosaponin C & 39.3 & 0.19 & Xian Mao \\
\hline MOL000449 & Stigmasterol & 43.8 & 0.76 & Xian Mao \\
\hline MOL001510 & 24-Epicampesterol & 37.6 & 0.71 & Xian Mao \\
\hline MOL001645 & Linoleyl acetate & 42.1 & 0.2 & $\begin{array}{l}\text { Yin yang } \\
\text { huo }\end{array}$ \\
\hline MOL001771 & Poriferast-5-en-3beta-ol & 36.9 & 0.75 & $\begin{array}{l}\text { Yin yang } \\
\text { huo }\end{array}$ \\
\hline MOL001792 & DFV & 32.8 & 0.18 & $\begin{array}{c}\text { Yin yang } \\
\text { huo }\end{array}$ \\
\hline MOL003044 & Chryseriol & 35.9 & 0.27 & $\begin{array}{l}\text { Yin yang } \\
\text { huo }\end{array}$ \\
\hline MOL003542 & 8-Isopentenyl-kaempferol & 38 & 0.39 & $\begin{array}{c}\text { Yin yang } \\
\text { huo }\end{array}$ \\
\hline MOL000359 & Sitosterol & 36.9 & 0.75 & $\begin{array}{l}\text { Yin yang } \\
\text { huo }\end{array}$ \\
\hline
\end{tabular}


TABle 1: Continued.

\begin{tabular}{|c|c|c|c|c|}
\hline Mol ID & Components & $\mathrm{OB} \%$ & DL & Herbs \\
\hline MOL000422 & Kaempferol & 41.9 & 0.24 & $\begin{array}{l}\text { Yin yang } \\
\text { huo }\end{array}$ \\
\hline MOL004367 & Olivil & 62.2 & 0.41 & $\begin{array}{l}\text { Yin yang } \\
\text { huo }\end{array}$ \\
\hline MOL004373 & Anhydroicaritin & 45.4 & 0.44 & $\begin{array}{l}\text { Yin yang } \\
\text { huo }\end{array}$ \\
\hline MOL004380 & C-Homoerythrinan,1,6-didehydro-3,15,16-trimethoxy-, (3. Beta)- & 39.1 & 0.49 & $\begin{array}{l}\text { Yin yang } \\
\text { huo }\end{array}$ \\
\hline MOL004382 & Yin yang huo A & 57 & 0.77 & $\begin{array}{l}\text { Yin yang } \\
\text { huo }\end{array}$ \\
\hline MOL004384 & Yin yang huo $\mathrm{C}$ & 45.7 & 0.5 & $\begin{array}{l}\text { Yin yang } \\
\text { huo }\end{array}$ \\
\hline MOL004386 & Yin yang huo $\mathrm{E}$ & 51.6 & 0.55 & $\begin{array}{l}\text { Yin yang } \\
\text { huo }\end{array}$ \\
\hline MOL004388 & $\begin{array}{l}\text { 6-Hydroxy-11,12-dimethoxy-2,2-dimethyl-1,8-dioxo-2,3,4,8-tetrahydro-1h-isochromeno[3,4- } \\
\text { h] isoquinolin-2-ium }\end{array}$ & 60.6 & 0.66 & $\begin{array}{l}\text { Yin yang } \\
\text { huo }\end{array}$ \\
\hline MOL004391 & 8-(3-Methylbut-2-enyl)-2-phenyl-chromone & 48.5 & 0.25 & $\begin{array}{l}\text { Yin yang } \\
\text { huo }\end{array}$ \\
\hline MOL004394 & Anhydroicaritin-3-O-alpha-L-rhamnoside & 41.6 & 0.61 & $\begin{array}{l}\text { Yin yang } \\
\text { huo }\end{array}$ \\
\hline MOL004396 & 1,2-bis(4-hydroxy-3-methoxyphenyl) propan-1,3-diol & 52.3 & 0.22 & $\begin{array}{l}\text { Yin yang } \\
\text { huo }\end{array}$ \\
\hline MOL004425 & Icariin & 41.6 & 0.61 & $\begin{array}{l}\text { Yin yang } \\
\text { huo }\end{array}$ \\
\hline MOL004427 & Icariside A7 & 31.9 & 0.86 & $\begin{array}{l}\text { Yin yang } \\
\text { huo }\end{array}$ \\
\hline MOL000006 & Luteolin & 36.2 & 0.25 & $\begin{array}{c}\text { Yin yang } \\
\text { huo }\end{array}$ \\
\hline MOL000622 & Magnograndiolide & 63.7 & 0.19 & $\begin{array}{c}\text { Yin yang } \\
\text { huo }\end{array}$ \\
\hline MOL000098 & Quercetin & 46.4 & 0.28 & $\begin{array}{c}\text { Yin yang } \\
\text { huo }\end{array}$ \\
\hline MOL001677 & Asperglaucide & 58 & 0.52 & Zhi mu \\
\hline MOL001944 & Marmesin & 50.3 & 0.18 & Zhi mu \\
\hline MOL003773 & Mangiferolic acid & 36.2 & 0.84 & Zhi mu \\
\hline MOL000422 & Kaempferol & 41.9 & 0.24 & Zhi mu \\
\hline MOL004373 & Anhydroicaritin & 45.4 & 0.44 & Zhi mu \\
\hline MOL004489 & Anemarsaponin F_qt & 60.1 & 0.79 & Zhi mu \\
\hline MOL004492 & Chrysanthemaxanthin & 38.7 & 0.58 & Zhi mu \\
\hline MOL004497 & Hippeastrine & 51.7 & 0.62 & Zhi mu \\
\hline MOL004514 & Timosaponin B III_qt & 35.3 & 0.87 & Zhi mu \\
\hline MOL000449 & Stigmasterol & 43.8 & 0.76 & Zhi mu \\
\hline MOL004528 & Icariin I & 41.6 & 0.61 & Zhi mu \\
\hline MOL004540 & Anemarsaponin C_qt & 35.5 & 0.87 & Zhi mu \\
\hline MOL004542 & Anemarsaponin E_qt & 30.7 & 0.86 & Zhi mu \\
\hline MOL000483 & (Z)-3-(4-hydroxy-3-methoxy-phenyl)-N-[2-(4-hydroxyphenyl) ethyl] acrylamide & 118 & 0.26 & Zhi mu \\
\hline MOL000546 & Diosgenin & 80.9 & 0.81 & Zhi mu \\
\hline MOL000631 & Coumaroyltyramine & 113 & 0.2 & Zhi mu \\
\hline MOL007107 & $\mathrm{C} 09092$ & 36.07 & 0.25 & Dan shen \\
\hline MOL007108 & Isocryptotanshi-none & 54.98 & 0.39 & Dan shen \\
\hline MOL007111 & Isotanshinone II & 49.92 & 0.4 & Dan shen \\
\hline MOL007115 & Manool & 45.04 & 0.2 & Dan shen \\
\hline MOL007118 & Microstegiol & 39.61 & 0.28 & Dan shen \\
\hline MOL007119 & Miltionone I & 49.68 & 0.32 & Dan shen \\
\hline MOL007120 & Miltionone II & 71.03 & 0.44 & Dan shen \\
\hline MOL007121 & Miltipolone & 36.56 & 0.37 & Dan shen \\
\hline MOL007122 & Miltirone & 38.76 & 0.25 & Dan shen \\
\hline MOL007123 & Miltirone II & 44.95 & 0.24 & Dan shen \\
\hline MOL007124 & Neocryptotanshinone ii & 39.46 & 0.23 & Dan shen \\
\hline MOL007125 & Neocryptotanshinone & 52.49 & 0.32 & Dan shen \\
\hline
\end{tabular}


TABle 1: Continued.

\begin{tabular}{|c|c|c|c|c|}
\hline Mol ID & Components & $\mathrm{OB} \%$ & DL & Herbs \\
\hline MOL007127 & 1-Methyl-8,9-dihydro-7h-naphtho[5,6-g] benzofuran-6,10,11-trione & 34.72 & 0.37 & Dan shen \\
\hline MOL007130 & Prolithospermic acid & 64.37 & 0.31 & Dan shen \\
\hline MOL007132 & (2R)-3-(3,4-dihydroxyphenyl)-2-[(Z)-3-(3,4-dihydroxyphenyl) acryloyl] oxy-propionic acid & 109.38 & 0.35 & Dan shen \\
\hline MOL007140 & (Z)-3-[2-[(E)-2-(3,4-dihydroxyphenyl) vinyl]-3,4-dihydroxy-phenyl] acrylic acid & 88.54 & 0.26 & Dan shen \\
\hline MOL007141 & Salvianolic acid $\mathrm{g}$ & 45.56 & 0.61 & Dan shen \\
\hline MOL007142 & Salvianolic acid $\mathrm{j}$ & 43.38 & 0.72 & Dan shen \\
\hline MOL007143 & Salvilenone I & 32.43 & 0.23 & Dan shen \\
\hline MOL007145 & Salviolone & 31.72 & 0.24 & Dan shen \\
\hline MOL007149 & NSC 122421 & 34.49 & 0.28 & Dan shen \\
\hline MOL007150 & $\begin{array}{c}\text { (6S)-6-hydroxy-1-methyl-6-methylol-8,9-dihydro-7h-naphtho[8,7-g] benzofuran-10,11- } \\
\text { quinone }\end{array}$ & 75.39 & 0.46 & Dan shen \\
\hline MOL007151 & Tanshindiol B & 42.67 & 0.45 & Dan shen \\
\hline MOL007152 & Przewaquinone $\mathrm{E}$ & 42.85 & 0.45 & Dan shen \\
\hline MOL007154 & Tanshinone iia & 49.89 & 0.4 & Dan shen \\
\hline MOL007155 & (6S)-6-(hydroxymethyl)-1,6-dimethyl-8,9-dihydro-7h-naphtho[8,7-g] benzofuran-10,11-dione & 65.26 & 0.45 & Dan shen \\
\hline MOL007156 & Tanshinone VI & 45.64 & 0.3 & Dan shen \\
\hline MOL002897 & Epiberberine & 43.09 & 0.78 & Huang lian \\
\hline MOL002903 & (R)-canadine & 55.37 & 0.77 & Huang lian \\
\hline MOL002904 & Berlambine & 36.68 & 0.82 & Huang lian \\
\hline MOL002907 & Corchoroside A_qt & 104.95 & 0.78 & Huang lian \\
\hline MOL000622 & Magnograndiolide & 63.71 & 0.19 & Huang lian \\
\hline MOL000762 & Palmidin A & 35.36 & 0.65 & Huang lian \\
\hline MOL000785 & Palmatine & 64.6 & 0.65 & Huang lian \\
\hline MOL000098 & Quercetin & 46.43 & 0.28 & Huang lian \\
\hline MOL001458 & Coptisine & 30.67 & 0.86 & Huang lian \\
\hline MOL002668 & Worenine & 45.83 & 0.87 & Huang lian \\
\hline MOL008647 & Moupinamide & 86.71 & 0.26 & Huang lian \\
\hline
\end{tabular}

eigenvector value was 0.039 . The top 10 compounds and top 6 key target of RSRN are shown in Table 2. The top six targets were PTGS2 $($ degree $=231)$, PTGS1 $($ degree $=148)$, SCN5A $($ degree $=137), \quad$ HSP90AA1 $\quad($ degree $=131), \quad$ NCOA2 $($ degree $=130)$, and ADRB2 $($ degree $=127)($ Figure $3(b)$ and $3(\mathrm{c})$ ).

3.3. Disease Targets Acquisition and Analysis. There were 1373 disease targets related to hyperlipidemia, 4481 targets related to atherosclerosis, and 9553 targets related to "hyperlipidemia," which were selected from GeneCards, TTD, DrugBank, and OMIM database. The above disease targets were analyzed by Venn map (Figure 4(a)). The data set GSE57691 was selected from the GEO database for screening differentially expressed genes. Then differential expression analysis on the data was performed using the limma software package. Compared with control samples, a total of 269 genes were significantly differentially expressed in hyperlipidemia samples, 47 were upregulated, and 221 were downregulated. The differentially expressed genes are shown in the cluster diagram (Figure 4(b)) and volcano map (Figure 4(c)). A total of 974 disease targets were obtained by merging above disease targets in the end.

3.4. Construction of Drug-Disease Target Network. After the construction of the Venn diagram, 106 targets between the 974 disease targets and 225 related targets of RSRN were selected as the potential targets in the treatment of hyperlipidemia-related diseases (Figure 5(a)). The protein interaction relationship was obtained by using BisoGenet plug-in of Cytoscape software (Figure 5(b)). Using the CytoNCA plug-in of Cytoscape software, the core diseasedrug targets was analyzed and confirmed. The selection criteria were set as follows: DC value $>61$ (Figure 5(c)) (yellow part in the figure was qualified), and $\mathrm{BC}$ value $>600$ (Figure 5(d)). The red rectangle in the topological network represents the last selected target genes, including AKT1, AR, NF- $\kappa$ B, CASP3, mTOR, ERBB2, CHUK, CAV1, MAPK8, MAPK14, HIF1A, PPARG, RELA, NR3C1, ESR2, FOS, CDK4, GSK3B, HSPB1, MYC, MDM2, EGFR, HSP90AA1, HSPA5, NOS2, ADRB2, VCAM1, APP, ESR1, XIAP, CASP8, Bax, ICAM1, SOD1.

3.5. Functional Enrichment Analysis. The 106 potential targets were then subjected to GO and KEGG analysis to explore the links between the functional units, their potential significance in the biological systems network. The GO terms were determined in the following categories (Figures 6(a) and 6(b)): 1900 biological processes (BP), 43 cellular components (CC), and 140 molecular functions (MF) branches. In the category $\mathrm{BP}$, the genes were associated with response to metal ion (GO:0010038), response to nutrient levels (GO: 0031667), response to lipopolysaccharide (GO:0032496), and response to molecular of bacterial origin (GO:0002237). In the category $\mathrm{CC}$, the genes were associated with cell components such as membrane raft (GO:0045121), membrane micro domain (GO:0098857), membrane region (GO: 0098589), and transcription regulator complex (GO: 


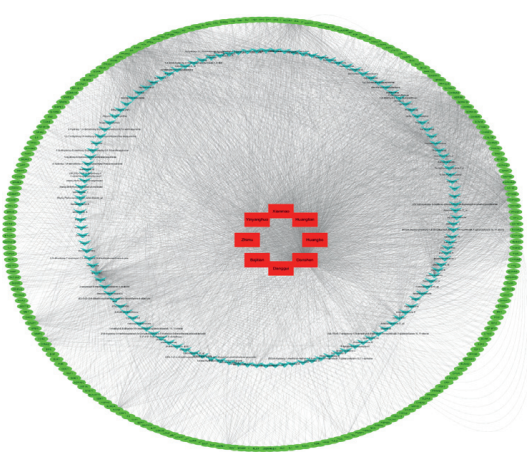

(a)

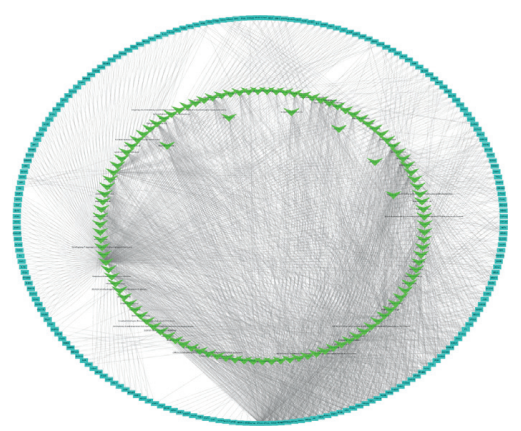

(b)

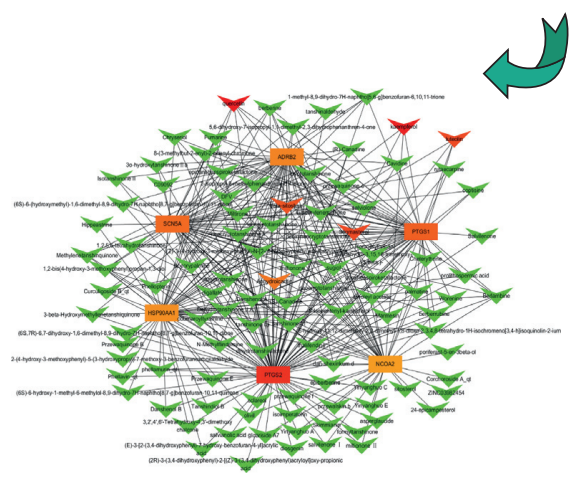

(c)

FIGURE 3: The "RSRN-compound-target" network and the key targets: (a) the RSRN-active component-target network, the red rectangle represents the name of drug, the blue inverted triangle represents the components, the green circle represents the targets; (b) the active component-key target network, the blue rectangle represents the key targets, the green inverted triangle represents the components.

TABLE 2: The active component and key target of RSRN.

\begin{tabular}{|c|c|c|c|c|c|}
\hline Compound & Type & Betweenness & Closeness & Degree & Eigenvector \\
\hline Quercetin & Mol & 6956.367 & 0.001616 & 426 & 0.172085 \\
\hline Kaempferol & Mol & 1074.972 & 0.001422 & 114 & 0.093268 \\
\hline Stigmasterol & Mol & 234.9962 & 0.001351 & 112 & 0.071736 \\
\hline Luteolin & Mol & 966.566 & 0.001414 & 110 & 0.077567 \\
\hline Beta-sitosterol & Mol & 188.0214 & 0.001361 & 88 & 0.076787 \\
\hline Anhydroicaritin & Mol & 173.2552 & 0.001372 & 66 & 0.083024 \\
\hline Danshinone II A & Mol & 1130.871 & 0.00122 & 41 & 0.073208 \\
\hline Dehydrotanshinone II A & Mol & 26.81371 & 0.001348 & 40 & 0.06437 \\
\hline Palmatine & Mol & 16.99011 & 0.001297 & 34 & 0.054885 \\
\hline C-homoerythrinan, 1,6-didehydro-3,15,16-trimethoxy-, (3. Beta.)- & Mol & 292.7947 & 0.001333 & 32 & 0.070996 \\
\hline PTGS2 & Gene & 6853.524 & 0.001597 & 231 & 0.158477 \\
\hline PTGS1 & Gene & 1835.112 & 0.001504 & 148 & 0.126258 \\
\hline SCN5A & Gene & 1382.629 & 0.001497 & 137 & 0.127246 \\
\hline HSP90AA1 & Gene & 2411.688 & 0.001497 & 131 & 0.107942 \\
\hline NCOA2 & Gene & 2395.628 & 0.001481 & 130 & 0.098189 \\
\hline ADRB2 & Gene & 1468.898 & 0.001495 & 127 & 0.12509 \\
\hline
\end{tabular}

0005667). In the category MF, the genes were associated with DNA binding transcription factor binding (GO:0140297) and RNA polymerase II specific DNA binding transcription factor binding (GO:0061629). The KEGG enrichment result indicated that the genes were associated with AGE-RAGE signaling pathway (hsa04933), fluid shear stress and atherosclerosis (hsa05418), PI3K-Akt signaling pathway (hsa04151), TNF signaling pathway (hsa04668), and NFkappa B signaling pathway (hsa04064) (Figures 6(c) and 6(d)). The KEGG network included 83 nodes and 360 edges (Figure 7(a)). The top six target genes were AKT1 (proteinserine-threonine kinase 1) (degree $=17)$, RELA (nuclear factor kappa B p65, NF- $\kappa$ B p65) (degree=16), CHUK (conserved helix-loop-helix ubiquitous kinase, also known as $\mathrm{I} \kappa \mathrm{B}$ kinase $\alpha$, IKK $\alpha$, or IKK1) (degree $=14)$, CCND1 (Cyclin D1) (degree=13), MAPK8 (mitogen-activated protein kinase 8$)($ degree $=12$ ), and MAPK14 (mitogenactivated protein kinase 14$)($ degree $=11)($ Figure $7(b))$. The 


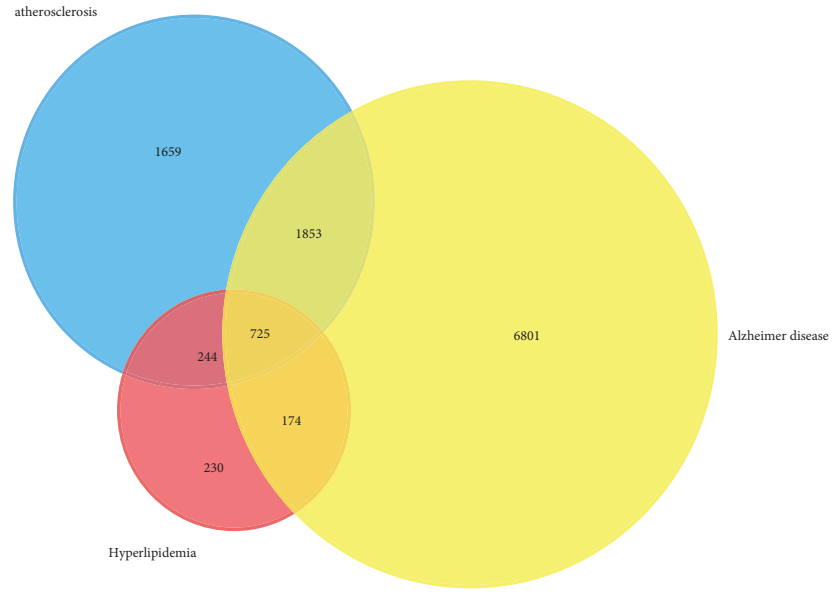

(a)

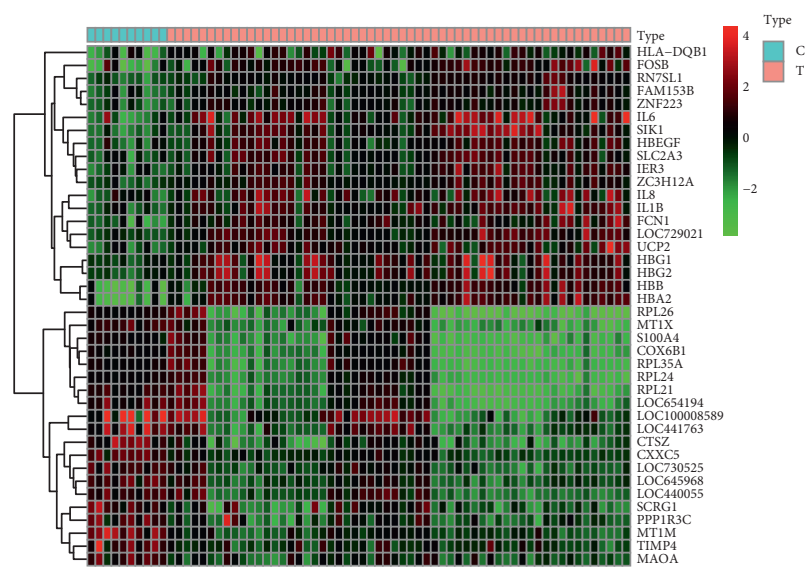

(b)

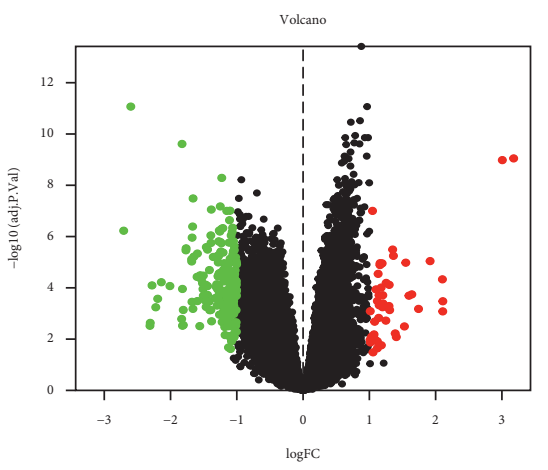

(c)

Figure 4: Disease targets analysis: (a) "hyperlipidemia," "atherosclerosis," and "Alzheimer's disease" were selected as the key words for the retrieval of disease targets; (b, c) the cluster diagram and volcano map of the differentially expressed genes in GSE57691 data set, red represents upregulated and green represents downregulated.

PI3K-Akt signaling pathway and NF-kappa B signaling pathway were performed by $R$ software (Figures 7 (c) and $7(d)$ ), and the red mark represents the potential target of RSRN intervention.

3.6. RSRN Downregulated the Expression of NF- $\kappa B p 65$ Protein in Hypothalamus of Ovariectomized Rats. The immunohistochemistry results showed that the positive expression of NF- $\kappa$ Bp 65 cells included glial cells, which were located in cytoplasm or nucleus (Figure 8(a)-8(e)). Compared with the SHAM group, the expression of NF- $\kappa$ Bp 65 protein in hypothalamus in OVX group was significantly increased $(p<0.05)$, with a large number of positive expression cells and dark brown color. Compared with the OVX group, the expression of NF- $\kappa$ Bp 65 protein in hypothalamus in RSRN group and EV group was significantly decreased $(p<0.05)$.

3.7. RSRN Regulated the Expression of NF- $\kappa B p 65, I \kappa B \alpha, T N F \alpha$, $I L-1 \beta$, and IL-6 in Brain of Ovariectomized Rats. Western blotting results showed that the expression levels of NF$\kappa \mathrm{Bp} 65$, TNF $\alpha$, IL- $1 \beta$, and IL- 6 in OVX group were significantly higher than those of the SHAM group $(p<0.05)$, while the expression of $\mathrm{I} \kappa \mathrm{B} \alpha$ was significantly decreased $(p<0.05)$. Compared with the OVX group, the protein expression levels of NF- $\kappa$ Bp $65, \mathrm{TNF} \alpha$, IL- $1 \beta$, and IL-6 in RSRN group were significantly decreased $(p<0.05)$, while the expression of $\mathrm{I} \kappa \mathrm{B} \alpha$ protein was significantly increased $(p<0.05)$ (Figure 9(a)-9(c); Figure 10(a)-10(c)).

\section{Discussion}

The method of reducing the south and reinforcing the north (RSRN) can nourish kidney essence and purging the heart. It is also called the method of purging fire and replenishing water. The method of RSRN in this study was used to coordinate yin and yang and to prevent postmenopausal-related diseases. There were 187 active components in RSRN, of which quercetin, kaempferol, Stigmasterol, luteolin, betasitosterol, and Anhydroicaritin were the main active components. Kaempferol and quercetin may have hypoglycemic, lipid-lowering, anti-inflammatory, antioxidant, and anticancer effects [17]. It was reported that Kaempferol can increase lipid metabolism by increasing $\operatorname{PPAR} \alpha$ level, decreasing SREBPs level, and promoting expression of ACO and CYP4A1, so as to reduce visceral fat accumulation and improve hyperlipidemia in obese rats fed with high-fat diet [18]. Quercetin can improve cholesterol reverse transport by upregulating the expression of ABCA1 and ABCG1 protein and enhancing the cholesterol acceptance of HDL and 


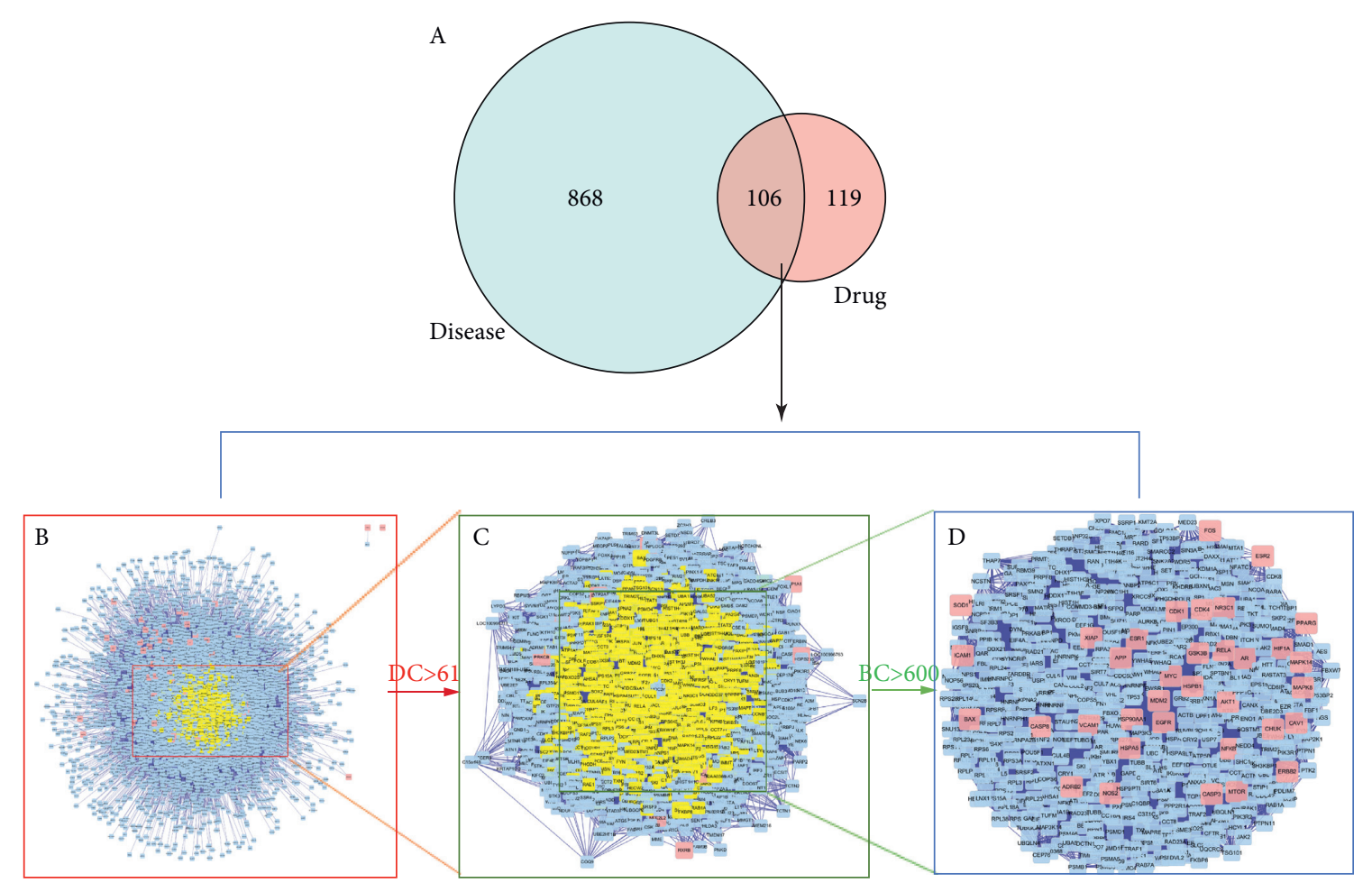

Figure 5: The drug-disease target network. (a) The Venn diagram for drug and disease targets. The overlap targets mean the potential therapeutic gene for RSRN when treating hyperlipidemia-related disease; (b) there were 6664 nodes and 146555 edges in the network; (c) the first screening threshold was DC >61, which resulted in 1350 nodes and 58513 edges; (d) the second screening threshold was BC $>600$, and 609 nodes and 25745 edges remained.

ApoA1 by reducing oxidation so as to reduce lipid accumulation [19]. Research [20] showed that luteolin could reduce the activation of PI3K/Akt induced by EGF and reduce the phosphorylation of EGFR, Akt, p38, and extracellular signal regulated kinase (ERK). Studies [21] found that pretreatment with luteolin could reduce the production of proinflammatory cytokines such as TNF- $\alpha$, IL-6, and inflammatory mediator nitric oxide (NO) which produced by lipopolysaccharide (LPS)-stimulated MH-S cells of mouse alveolar macrophages. Studies have illustrated that $\beta$ sitosterol exerts cholesterol-lowering, antioxidant, and antiinflammatory effects $[22,23]$. The above studies showed that the main active ingredients of RSRN had anti-inflammatory, antioxidation stress, hypoglycemic and lipid-lowering effects, and cardiovascular system protection.

A total of 106 drug-disease core targets were obtained, and the genes were used to constructed the core target gene topology network and the KEGG network. The top six target genes from the KEGG network were merged with the candidate genes from the core target gene topology network; we obtained the key target genes including AKT1, RELA, CHUK, MAPK8, MAPK14, and CCND1. The key targets are mainly involved in NF- $\kappa \mathrm{B} / \mathrm{MAPK}$ signaling pathway, PI3K-Akt signaling pathway, atherosclerosis, and inflammation-related signaling pathways. Akt is the central link of PI3K/Akt signaling pathway, and it plays an important role in regulating cell survival, protein synthesis, angiogenesis, and insulin-dependent metabolic cell response [24]. Akt can inhibit apoptosis, phosphorylate caspase-9 precursor $[25,26]$. Nuclear factor $\kappa \mathrm{B}(\mathrm{NF}-\kappa \mathrm{B})$ plays an important role in the regulation of gene transcription related to inflammation, cell proliferation, differentiation and apoptosis, immune response, and tumor formation; the human NF- $\kappa \mathrm{B}$ family consists of five members: P50/P105, p52/P100, p65/ RELA, RELB, and c-Rel, which are encoded by NF- $\kappa$ B1, NF$\kappa B 2$, RELA, RELB, and REL genes [27]. CHUK (also known as IKK $\alpha$, IKK $\alpha$ ) is the upstream component of signal transduction pathway that directly enters the nucleus to regulate gene expression, and it is also a component of activating cytokine protein complex, studies have shown that gene mutation of CHUK is associated with hypertension and lipid abnormality [28-30]. These studies indicated that the key targets have the functions of regulating lipid metabolism, glucose metabolism, and immune regulation, which were of great significance for the prevention and treatment of hyperlipidemia, and also had certain effects on the complications of atherosclerosis and Alzheimer's disease.

The core targets of RSRN-active ingredients in the treatment of hyperlipidemia-related diseases may involve in the response to lipopolysaccharide, oxidative stress, and DNA binding transcription factor binding. Oxidative stress plays an important role in the pathogenesis of atherosclerosis (AS), hypertension, metabolic syndrome, hypercholesterolemia, Alzheimer's disease, aging, and cancer [31]. Lipopolysaccharide (LPS) activates 


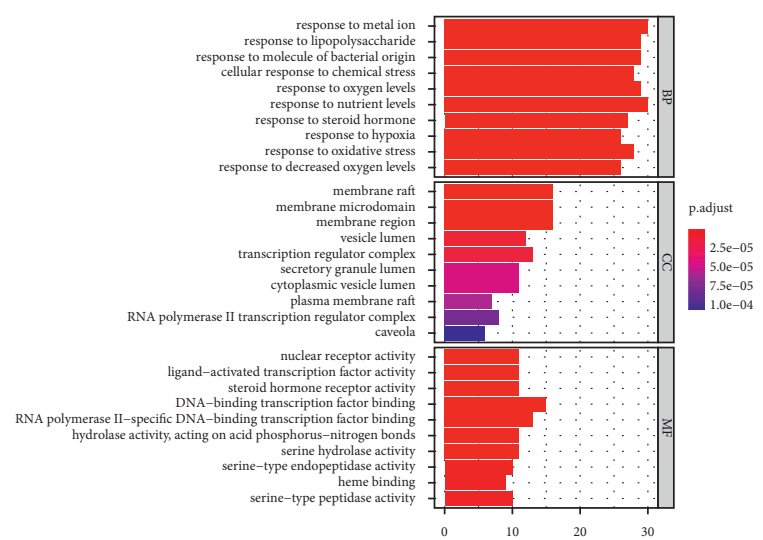

(a)

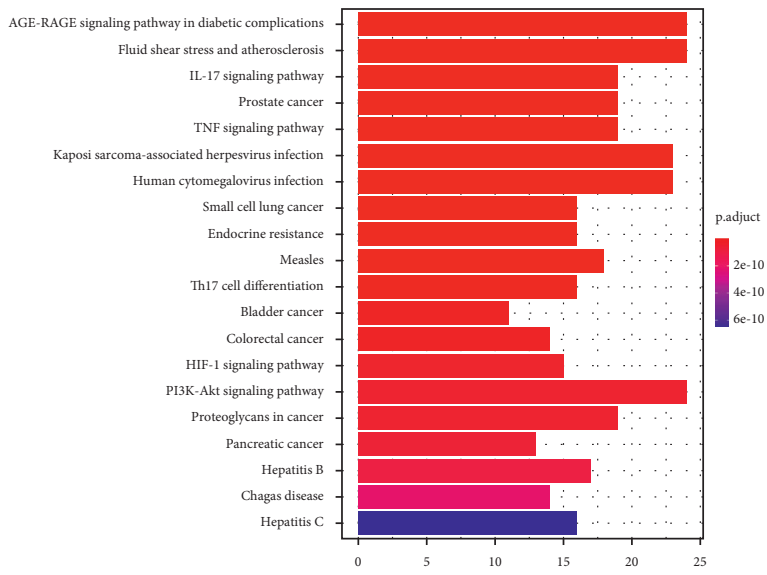

(c)

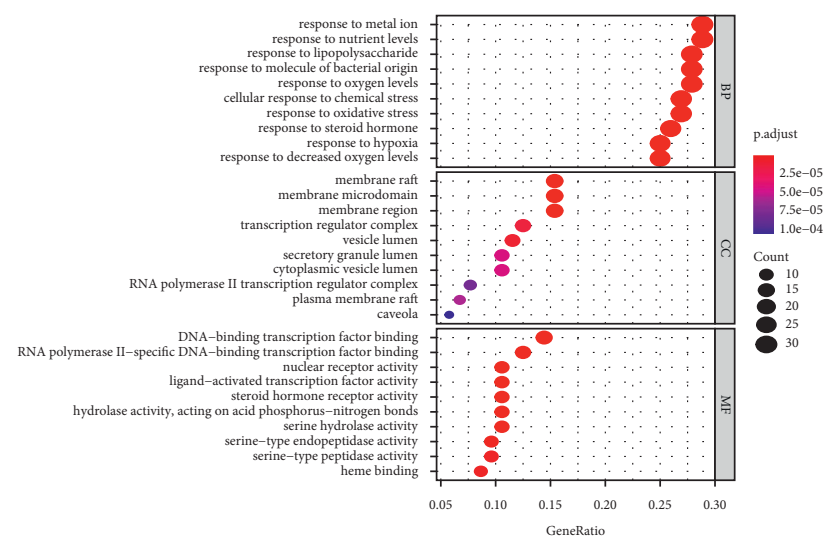

(b)

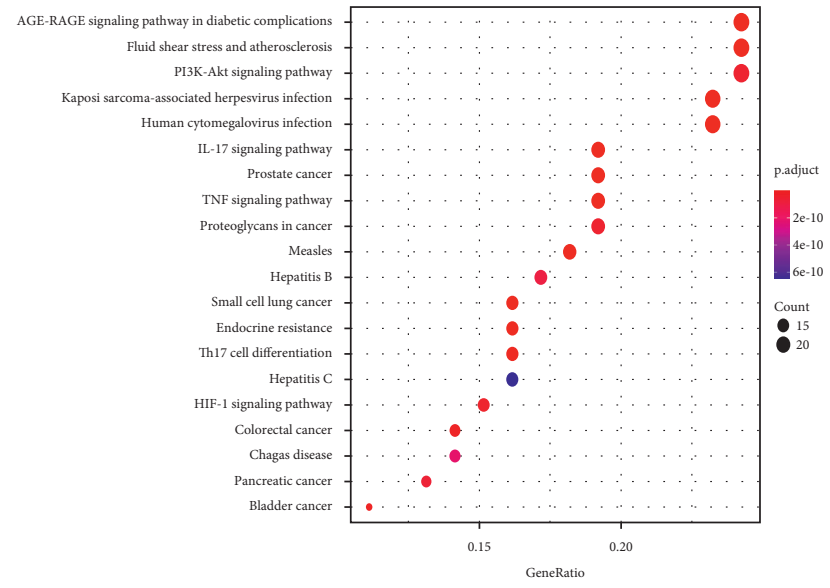

(d)

Figure 6: Functional enrichment analysis. (a, b) GO functional enrichment analysis; (c, d) KEGG functional enrichment analysis.

downstream target genes by activating TLR4/NF- $\kappa \mathrm{B}$ signaling pathways, thus to release inflammatory factors such as TNF- $\alpha$, IL-8, IL-1, and IL-6 [32]. Endotoxin is an inflammatory reaction promoter of LPS in the outer membrane of Gram-negative bacteria; it is the main ligand of Toll like receptor; and it has been confirmed that endotoxin plays an important role in the process and progress of AS [33, 34].

The main pathways of RSRN may be involved in AGERAGE signaling pathway, cell fluid shear stress and atherosclerosis, and PI3K-Akt signaling pathway. Advanced glycosylated compounds (AGEs) are complex compounds produced by nonenzymatic glycosylation and oxidation of proteins, lipids, and nucleic acids; they can activate AGE-RAGE signaling pathway, MAPK signaling pathway, and NF- $\kappa$ B signaling pathway, leading to the expression of proinflammatory cytokines such as IL-1, IL6 , and TNF- $\alpha$, and the release of VCAM1, VEGF, and RAGE, so as to promote the development of atherosclerosis [35]. Cell fluid shear stress and atherosclerotic pathway play an important role in the development of dyslipidemia to atherosclerosis; it has been reported that the wall concentration of lipid in the slow flow area was higher than that in the high-speed laminar flow area, and the action time of lipid and arterial wall was prolonged, which was easy to cause atherosclerosis, and it also can promote oxidative stress, increase the production of ox LDL, upregulate the expression of NF- $\kappa \mathrm{B}$, thus to promote inflammatory response [36, 37]. PI3K/Akt signaling pathway plays an important role in lipid metabolism and inflammation regulation; inhibition of PI3K/Akt signaling pathway can significantly reduce serum-free fatty acids, cholesterol, and triglyceride [38, 39], and it also inhibited the secretion of proinflammatory mediators such as TNF$\alpha$ and IL- $1 \beta$ [40]. It can be speculated that RSRN may regulate dyslipidemia and prevent or delay the occurrence and development of AS through regulation of endocrine, metabolic, and inflammatory pathways.

Studies have found that hyperlipidemia is closely related to hypothalamic inflammatory response. Hypothalamic inflammation leads to the occurrence of obesitybased metabolic diseases. A short-term high-fat diet can increase the expression of biomarkers and promote inflammatory response in the basal hypothalamus to form a transient inflammation. Under the condition of long-term high-fat diet, hypothalamic glial hyperplasia, and nerve injury can promote the occurrence of hypothalamic inflammation [41]. In this experimental study, the results showed that the expression of NF- $\kappa$ B p65, TNF- $\alpha$, IL- $1 \beta$, IL-6 in hypothalamic nucleus of OVX group was 


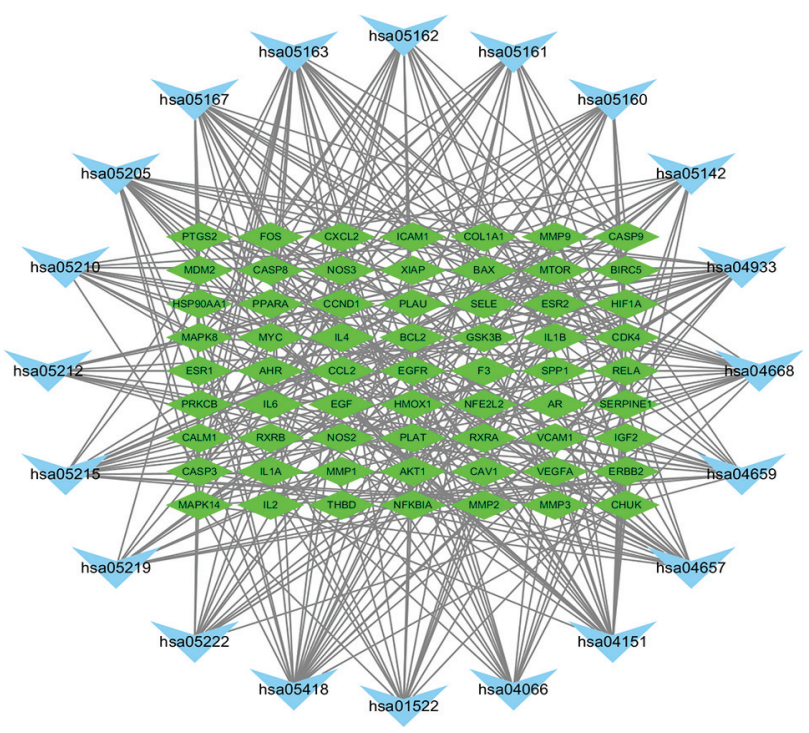

(a)

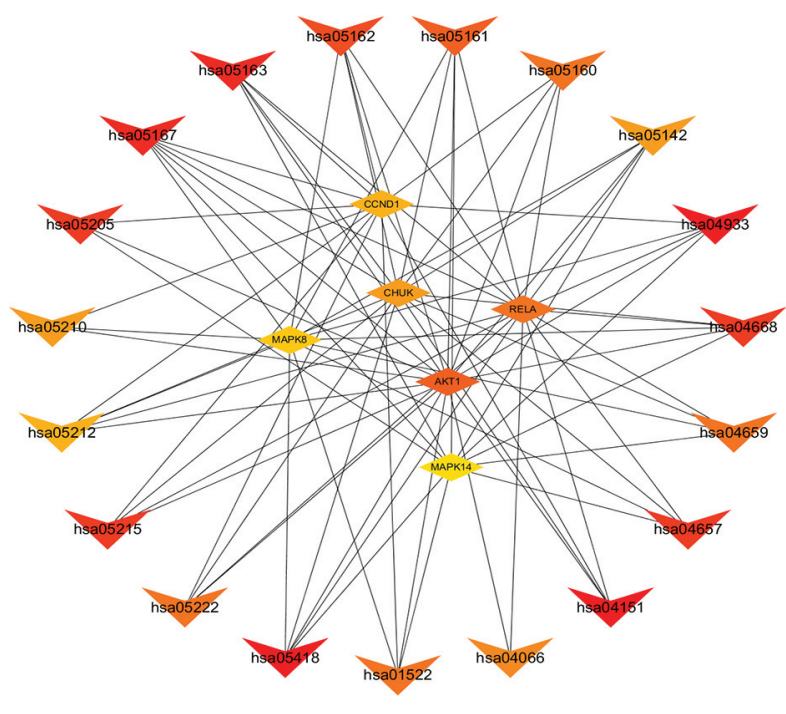

(b)

Figure 7: Continued.

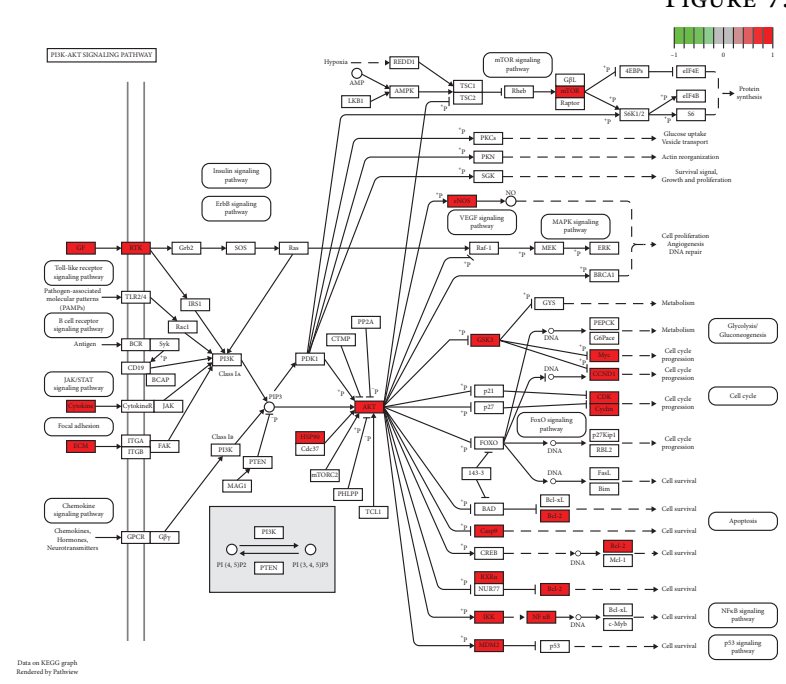

(c)

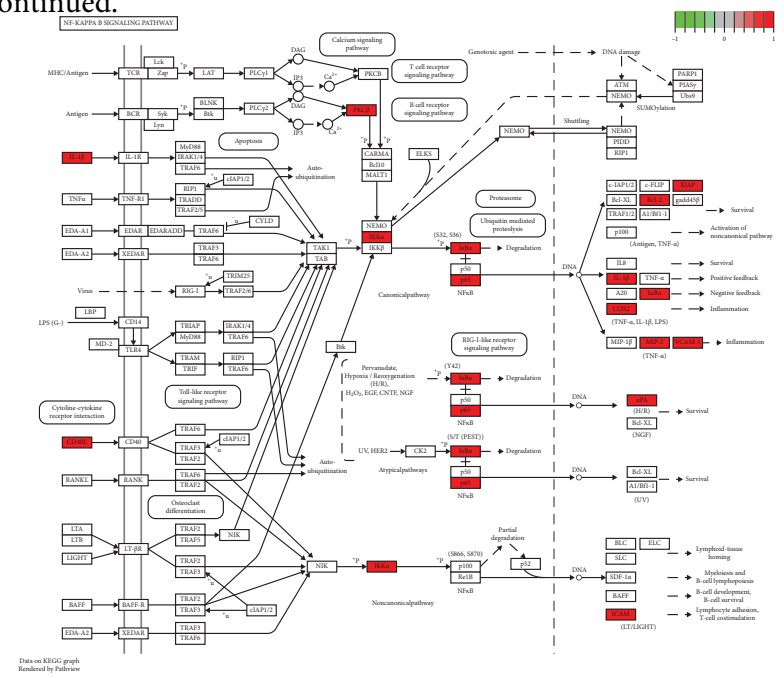

(d)

FIGURE 7: KEGG network and key pathways: (a) KEGG pathways target genes network, blue inverted triangle represents KEGG pathways, green rectangle represents KEGG pathway-related genes; (b) top 25 pathways and target genes; (c) PI3K-AKT signal pathway; (d) NFKAPPA B signal pathway.

significantly increased, which indicated that NF- $\kappa \mathrm{B}$ signal pathway and inflammatory cytokines were activated under the stimulation of intracellular and extracellular signals; after treatment with RSRN, the expression of activated NF- $\kappa$ B (p65) in nucleus was significantly reduced, and the expression of TNF- $\alpha$, IL- $1 \beta$, and IL- 6 was also significantly reduced in RSRN group; the results showed that RSRN could inhibit the activity of NF- $\kappa$ B and reduce the release of inflammatory cytokines. In the future, it is necessary to further explore the relationship between target protein and the upstream and downstream molecules of the signaling pathway and the specific regulatory mechanisms and confirm the curative effect through clinical trials. 


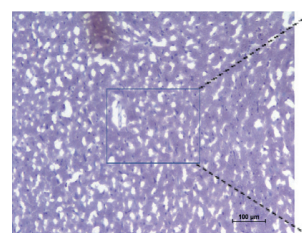

$\operatorname{SHAM}(100 \times)$

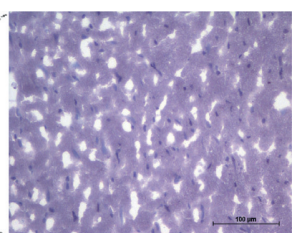

$\operatorname{SHAM}(200 \times)$

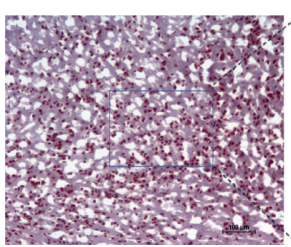

$\operatorname{OVX}(100 \times)$

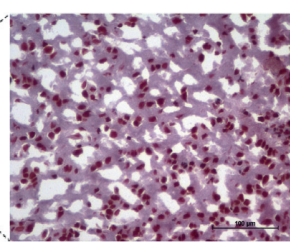

$\operatorname{OVX}(200 \times)$

(b)

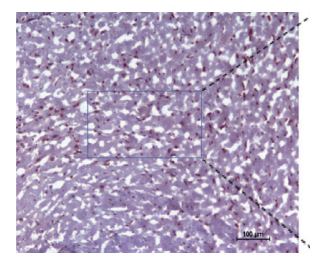

RSRN (100x) (a)

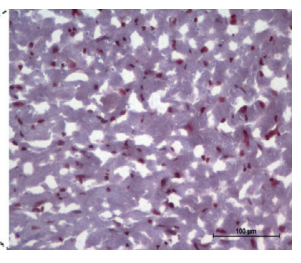

$\operatorname{RSRN}(200 \times)$

(c)

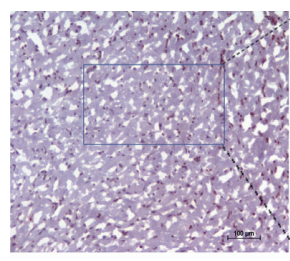

$\operatorname{EV}(100 \times)$

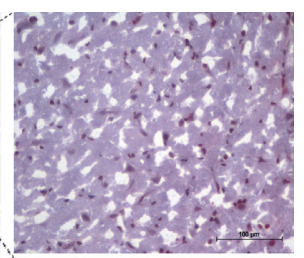

$\operatorname{EV}(200 \times)$

(d)

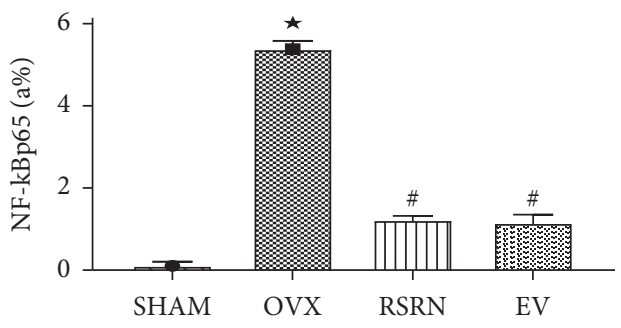

(e)

Figure 8: The expression of NF- $\kappa$ Bp65 in hypothalamus. (a-d) The expressions of NF-kBp65 in hypothalamus tissue were analyzed by immunohistochemistry $(\times 200)$. (e) Values are presented as the mean \pm standard deviation (SD), $n=3$ per group. ${ }^{\star} p<0.05$, compared with the SHAM group; ${ }^{\#} p<0.05$, compared with the OVX group.

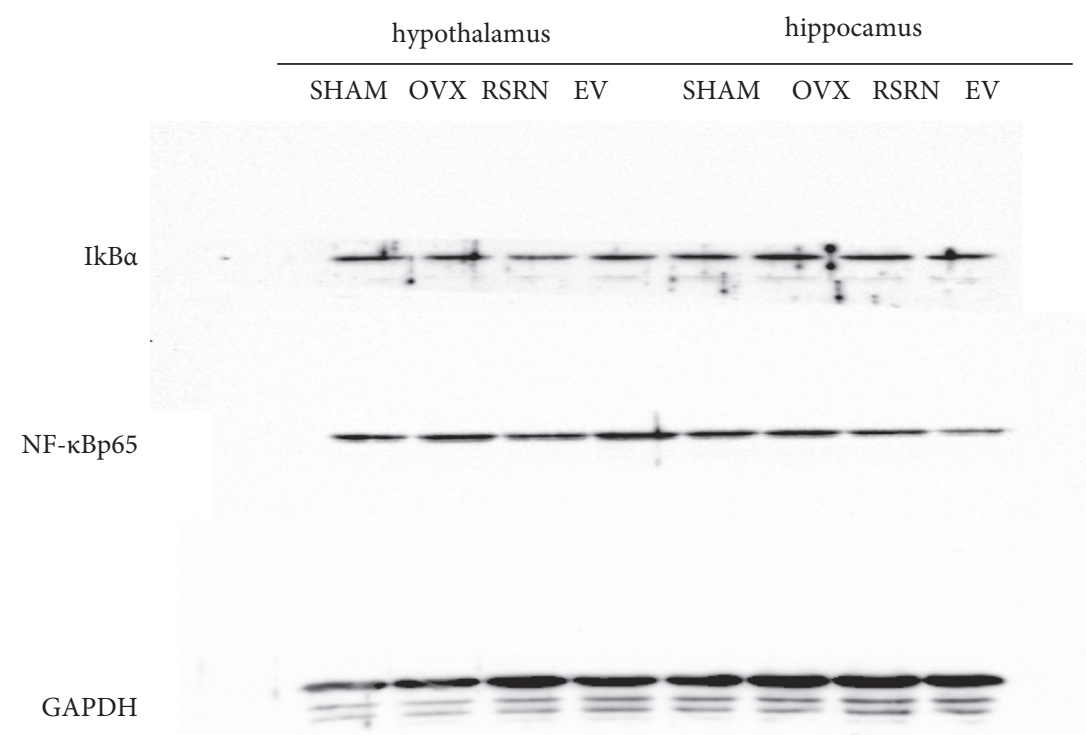

(a)

Figure 9: Continued. 


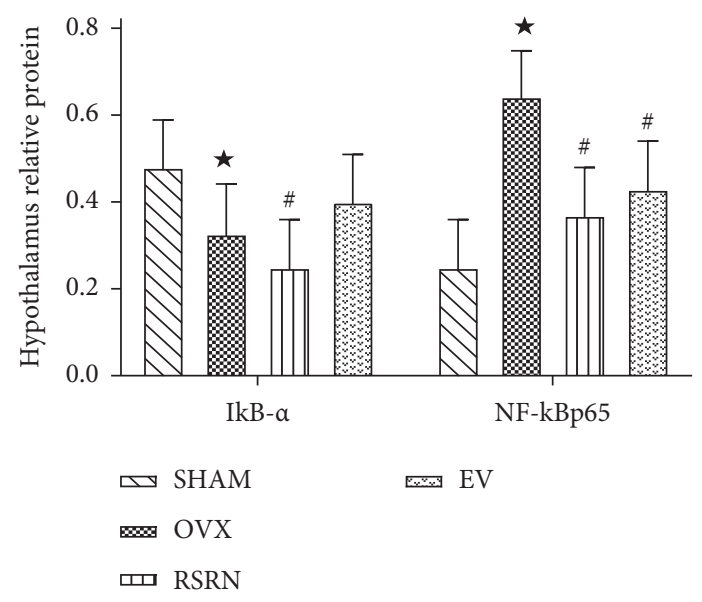

(b)

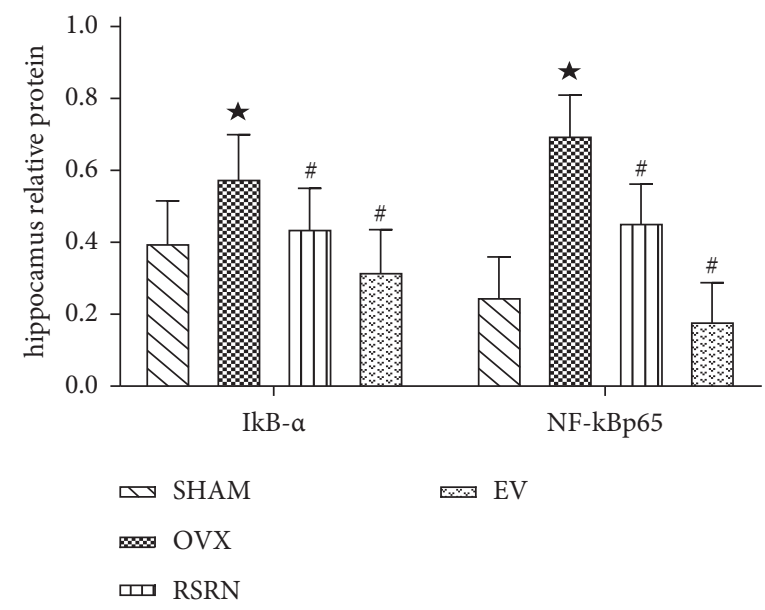

(c)

Figure 9: Protein expression of $\mathrm{I} \kappa \mathrm{B} \alpha$ and NF- $\kappa \mathrm{Bp} 65$ by western blot: (a) gene levels of NF- $\kappa \mathrm{Bp} 65, \mathrm{I} \kappa \mathrm{B} \alpha$ in hypothalamus and hippocampus by western blot; (b) semi-quantitative analysis of NF- $\kappa \mathrm{Bp} 65, \mathrm{I} \kappa \mathrm{B} \alpha$ proteins expression compared with GAPDH. Values are presented as the means \pm standard deviation (SD), $n=3$ per group. ${ }^{\star} p<0.05$, compared with the SHAM group; ${ }^{\#} p<0.05$, compared with the OVX group.

\begin{tabular}{ccc} 
hypothalamus & \multicolumn{3}{c}{ hippocamus } \\
\hline SHAM OVX RSRN EV & SHAM & OVX RSRN EV
\end{tabular}

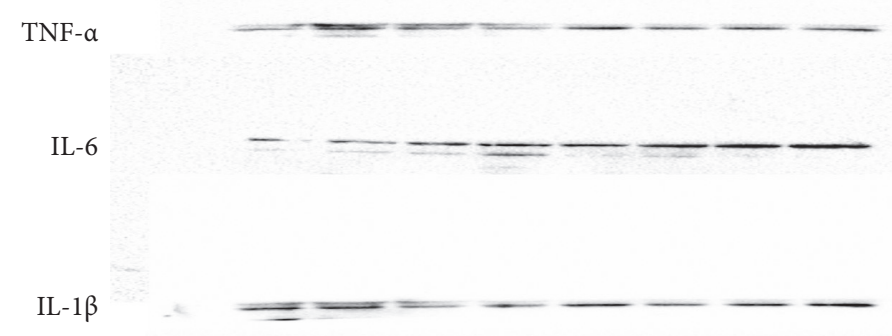




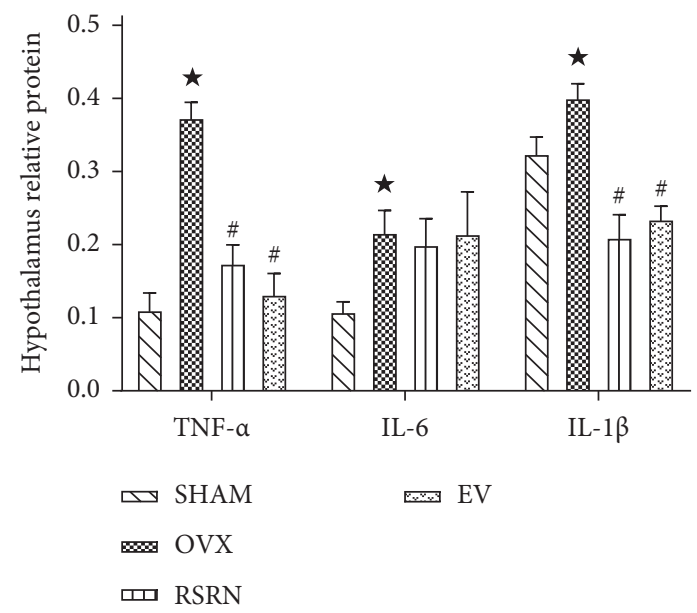

(b)

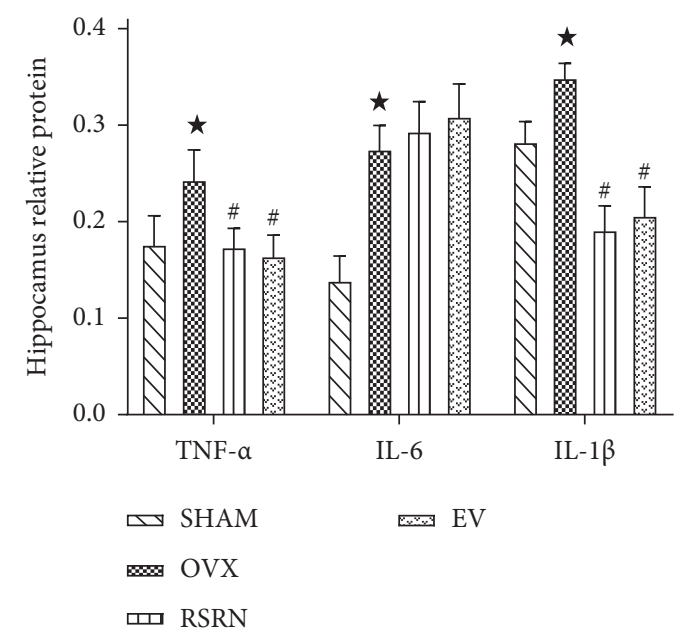

(c)

Figure 10: Protein expression of TNF $\alpha$, IL-1 $\beta$, and IL-6 by western blot: (a) gene levels of TNF $\alpha$, IL-1 $\beta$, and IL-6 in hypothalamus and hippocampus by western blot; (b) semi-quantitative analysis of TNF $\alpha$, IL- $1 \beta$, and IL- 6 proteins expression compared with GAPDH. Values are presented as the means \pm standard deviation (SD), $n=3$ per group. ${ }^{\star} p<0.05$, compared with the SHAM group; ${ }^{*} p<0.05$, compared with the OVX group.

\section{Conclusion}

In this study, a total of 187 potential active components and 106 related core targets were obtained and identified overall. Then after the Metascape enrichment analysis, RSRN may regulate AKT1, NF- $\kappa$ Bp65, IKK $\alpha$, TNF- $\alpha$, IL-1 $\beta$, IL-6 through TNF signaling pathway, PI3K-Akt signaling pathway, and NF-kappa B signaling pathway, so as to regulate lipid metabolism, inflammatory response, and prevent or delay the development of atherosclerotic diseases. This study suggests that RSRN may be used in the treatment of hyperlipidemia and related diseases. Due to the limitation of database data and corresponding analysis algorithms, the results may be biased, and further in vitro and in vivo studies are needed to verify the results.

\section{Abbreviations}

ADME: Absorption, distribution, metabolism, and excretion
OB: Oral bioavailability

DL: Drug-likeness

GO: $\quad$ Gene ontology

KEGG: Kyoto encyclopedia of genes and genomes

CC: $\quad$ Cellular components

MF: $\quad$ Molecular functions

BP: Biological processes

TCMSP: Traditional Chinese medicine systems pharmacology database and analysis platform.

\section{Data Availability}

All the data generated or analyzed during this study are included within the paper.

\section{Conflicts of Interest}

The authors declare that they have no conflicts of interest. 


\section{Authors' Contributions}

Hongjin $\mathrm{Wu}$ conceived and designed the experiment. Weiwei Dai analyzed the data and edited the manuscript. Jie Zhang, Libo Wang, and Chenglong Wang were responsible for figure drawing and table design and the manuscript revising. All the authors have read and approved the final manuscript.

\section{Acknowledgments}

This study was supported by grant from Natural Science Foundation of Shanghai, China (Project no. 16ZR1447300).

\section{References}

[1] W. Liu, S. Fu, and G. W. Fan, "Treatment progress of dyslipidemia in postmenopausal women," Chinese Journal of Gerontology, vol. 36, no. 16, p. 4110, 2016.

[2] N. Wang, P. Chen, Y. F. Hong, J. Sun, Y. Qin, and C. H. Du, "Progress of hyperlipidemia treated by traditional Chinese Medicine," Journal of Practical traditional Chinese Medicine, vol. 35, no. 2, pp. 247-249, 2019.

[3] Y. Li, K. H. Sun, F. Bai, H. M. Luo, and Z. Z. Wu, "Progress on pathogenesis of hyperlipidemia related diseases," Journal of Liaoning University of Traditional Chinese Medicine, vol. 21, no. 4, pp. 84-87, 2019.

[4] Y. Z. Zhou and J. Q. Lu, "Progress in the treatment of atherosclerosis with Chinese Medicine," Hunan Journal of Traditional Chinese medicine, vol. 33, no. 4, pp. 169-171, 2017.

[5] X. Qi, "Probe and analysis on pathogenic mechanism of climacterium cobined with hyperlipemia," Jilin Journal of Traditional Chinese Medicine, vol. 27, no. 1, pp. 4-6, 2007.

[6] H. Wu, D. Wei-wei, L. Wang, J. Zhang, and C. Wang, "Effects of reducing the south and reinforcing the north method on sex hormones and lipid metabolism of ovariectomized female rats," SCJTCMP, vol. 33, no. 9, pp. 4114-4117, 2018.

[7] H. J. Wu, Z. F. Zhang, J. T. Xu, T. T. Zhang, L. W. Xu, and S. G. Li, "Changes of pulse diagram parameter and sex hormone level before and after the Chinese medicine treatment in perimenopausal syndrome with syndrome of yin deficiency of liver and kidney," CJTCMP, vol. 32, no. 4, pp. 1870-1873, 2017.

[8] Y. H. Jiang, Y. H. Wang, Y. J. Liu, L. H. Xiang, Z. G. Zhang, and Y. J. Chen, "Effect of erxian decoction on myocardial microvessels and hemorheology in ovariectomized rats," Chinese journal of experimental Traditional Medical formulae, vol. 26, no. 24, pp. 59-67, 2020.

[9] Y. H. Wang, Changes of Cardiac Function and Glucose and Lipid Metabolism in Ovariectomized Rats and Intervention of Erxian Tang, Chinese Academy of traditional Chinese Medicine, Beijing, China, 2020.

[10] G. Chen, C. Huang, Y. Liu et al., "A network pharmacology approach to uncover the potential mechanism of yinchensini decoction," Evidence-based Complementary and Alternative Medicine, vol. 2018, Article ID 2178610, 14 pages, 2018.

[11] J. Ru, P. Li, J. Wang et al., "TCMSP: a database of systems pharmacology for drug discovery from herbal medicines," Journal of Cheminformatics, vol. 6, no. 1, p. 13, 2014.

[12] X. Shen, Z. Zhao, H. Wang, Z. Guo, B. Hu, and G. Zhang, "Elucidation of the anti-inflammatory mechanisms of Bupleuri and Scutellariae radix using system pharmacological analyses," Mediators of Inflammation, vol. 2017, Article ID 3709874, 10 pages, 2017.

[13] N. Wu, X. K. Mao, W. Wei, N. Yu, Z. Y. Rao, and H. S. Li, "Comparative study on the molecular mechanism of the treatment of ulcerative colitis by Four prescription for recuperating intestines based on network pharmacology and molecular docking," Journal of Chinese Medicinal Materials, vol. 1, pp. 186-192, 2020.

[14] Y. Yao, Y. Wu, Z. Ma, Y. Y. Zhang, Y. Li, and H. M. Bian, "Effect of Liuwei Dihuang Formula and its compatibility on rats with ovariectomy-induced atherosclerosis," Chinese Traditional Patent Medicine, vol. 38, no. 10, pp. 2111-2117, 2016.

[15] H. C. Ni, J. Li, Y. Jin, H. M. Zang, and L. Peng, "The experimental animal model of hyperlipidemia and hyperlipidemic fatty liver in rats," Chinese Pharmacological Bulletin, vol. 20, no. 6, pp. 703-706, 2004.

[16] Q. Sun, W. Zhong, W. Zhang, and Z. Zhou, "Defect of mitochondrial respiratory chain is a mechanism of ROS overproduction in a rat model of alcoholic liver disease: role of zinc deficiency," American Journal of Physiology - Gastrointestinal and Liver Physiology, vol. 310, no. 3, pp. G205-G214, 2016.

[17] M. Imran, A. Rauf, Z. A. Shah et al., "Chemo-preventive and therapeutic effect of the dietary flavonoid kaempferol: a comprehensive review," Phytotherapy Research, vol. 33, no. 2, pp. 263-275, 2019.

[18] C. Chang, T.-F. Tzeng, S.-S. Liou, Y.-S. Chang, and I.-M. Liu, "Kaempferol regulates the lipid-profile in high-fat diet-fed rats through an increase in hepatic PPAR $\alpha$ Levels," Planta Medica, vol. 77, no. 17, pp. 1876-1882, 2011.

[19] Y. Cui, P. Hou, F. Li et al., "Quercetin improves macrophage reverse cholesterol transport in apolipoprotein E-deficient mice fed a high-fat diet," Lipids in Health and Disease, vol. 16, no. 1, p. 9, 2017.

[20] E.-J. Lee, S.-Y. Oh, and M.-K. Sung, "Luteolin exerts antitumor activity through the suppression of epidermal growth factor receptor-mediated pathway in MDA-MB-231 ERnegative breast cancer cells," Food and Chemical Toxicology, vol. 50, no. 11, pp. 4136-4143, 2012.

[21] C.-Y. Chen, W.-H. Peng, K.-D. Tsai, and S.-L. Hsu, "Luteolin suppresses inflammation-associated gene expression by blocking NF- $\kappa \mathrm{B}$ and AP- 1 activation pathway in mouse alveolar macrophages," Life Sciences, vol. 81, no. 23-24, pp. 1602-1614, 2007.

[22] H. S. Wong, N. Chen, P. K. Leong, and K. M. Ko, “ $\beta$-Sitosterol enhances cellular glutathione redox cycling by reactive oxygen species generated from mitochondrial respiration: protection against oxidant injury in $\mathrm{H} 9 \mathrm{c} 2$ cells and rat hearts," Phytotherapy Research, vol. 28, no. 7, pp. 999-1006, 2014.

[23] I. Lampronti, M. C. Dechecchi, A. Rimessi et al., " $\beta$-Sitosterol reduces the expression of chemotactic cytokine genes in cystic fibrosis bronchial epithelial cells," Frontiers in Pharmacology, vol. 8, p. 236, 2017.

[24] K. M. Vasudevan and L. A. Garraway, "Akt signaling in physiology and disease," Current Topics in Microbiology and Immunology, vol. 347, no. 1, pp. 105-133, 2010.

[25] J.-H. Quan, G.-H. Cha, W. Zhou, J.-Q. Chu, Y. Nishikawa, and Y.-H. Lee, "Involvement of PI 3 kinase/Akt-dependent Bad phosphorylation in Toxoplasma gondii-mediated inhibition of host cell apoptosis," Experimental Parasitology, vol. 133, no. 4, pp. 462-471, 2013.

[26] T. Ngoc, M. A. Park, C. S. Jacqueline et al., "HnRNP U enhances caspase- 9 splicing and is modulated by AKT- 
dependent phosphorylation of HnRNP L," Journal of Biological Chemistry, vol. 288, no. 12, pp. 8575-8584, 2013.

[27] L. Yang, J. Deng, and Z. H. Deng, "Progress of NF- $\kappa$ B and its gene polymorphism in relation to inflammation and tumor," Guizhou Medical Journal, vol. 40, no. 10, pp. 1098-1099, 2016.

[28] J. Basson, L. de Las Fuentes, and D..C. Rao, "Single nucleotide polymorphism-single nucleotide polymorphism interactions among inflammation genes in the genetic architecture of blood pressure in the framingham heart study," American Journal of Hypertension, vol. 28, no. 2, pp. 248-255, 2015.

[29] F. W. Asselbergs, Y. Guo, E. P. van Iperen et al., "Large-scale gene-centric meta-analysis across 32 studies identifies multiple lipid loci," The American Journal of Human Genetics, vol. 91, no. 5, pp. 823-838, 2012.

[30] Q. G. Wei, Y. L. Wu, Z. W. Chen, J. Y. Zhou, J. J. Xie, and L. Gu, "Influence of Helix-loop-helix domain diffusion kinase (CHUK) gene polymorphism on lipid metabolism of Ischemic stroke and phlegm stasis resistance syndrome," Yunnan zhongyi Zhongyao Zazhi, vol. 36, no. 12, pp. 61-64, 2015.

[31] P. D. Ray, B.-W. Huang, and Y. Tsuji, "Reactive oxygen species (ROS) homeostasis and redox regulation in cellular signaling," Cellular Signalling, vol. 24, no. 5, pp. 981-990, 2012.

[32] W. Wang, Z. Y. Liu, and G. R. Chen, "Progress in studies of endotoxin receptor and anti-endotoxin," Chinese archives of traditional Chinese Medicine, vol. 34, no. 10, pp. 2367-2370, 2016.

[33] C. Hayashi, A. G. Madrigal, X. Liu et al., "Pathogen-mediated inflammatory atherosclerosis is mediated in part via Toll-like receptor 2-induced inflammatory responses," Journal of Innate Immunity, vol. 2, no. 4, pp. 334-343, 2010.

[34] K. Unkelbach, A. Gardemann, M. Kostrzewa, M. Philipp, H. Tillmanns, and W. Haberbosch, "A new promoter polymorphism in the gene of lipopolysaccharide receptor CD14 is associated with expired myocardial infarction in patients with low atherosclerotic risk profile," Arteriosclerosis, Thrombosis, and Vascular Biology, vol. 19, no. 4, pp. 932-938, 1999.

[35] Z. Hegab, S. Gibbons, N. Ludwig, and M. A. Mamas, "Role of advanced glycation end products in cardiovascular disease," World Journal of Cardiology, vol. 4, no. 4, pp. 90-102, 2012.

[36] R. D. Simmons, S. Kumar, and H. Jo, "The role of endothelial mechanosensitive genes in atherosclerosis and omics approaches," Archives of Biochemistry and Biophysics, vol. 591, pp. 111-131, 2016.

[37] J. Adam, Z. Teng, P. C. Evans, J. H. Gillard, H. Samady, and M. R. Bennett, "Role of biomechanical forces in the natural history of coronary atherosclerosis," Nature Reviews Cardiology, vol. 13, no. 4, pp. 210-220, 2016.

[38] V. R. Sopasakis, P. Liu, R. Suzuki et al., "Specific roles of the p110alpha isoform of phosphatidylinsositol 3-kinase in hepatic insulin signaling and metabolic regulation," Cell Metabolism, vol. 11, no. 3, pp. 220-230, 2010.

[39] C. M. Taniguchi, T. Kondo, M. Sajan et al., "Divergent regulation of hepatic glucose and lipid metabolism by phosphoinositide 3-kinase via Akt and $\mathrm{PKC} \lambda / \zeta$," Cell Metabolism, vol. 3, no. 5, pp. 343-353, 2006.

[40] S. Xie, M. Chen, B. Yan, X. He, X. Chen, and D. Li, "Identification of a role for the PI3K/AKT/mTOR signaling pathway in innate immune cells," PloS One, vol. 9, no. 4, Article ID e94496, 2014.

[41] Y. Gong, X. Shi, J. Niu et al., "Hypothalamic inflammation and mechanism of pathogenesis for obesity," Journal of Liaoning University of TCM, vol. 16, no. 7, pp. 85-88, 2014. 